\title{
UV camera conceptual designs for TMT Fiber WFOS
}

L. Gilles, D. Shafer, B. Delabre

L. Gilles, D. Shafer, B. Delabre, "UV camera conceptual designs for TMT Fiber WFOS," Proc. SPIE 10702, Ground-based and Airborne Instrumentation for Astronomy VII, 10702AD (13 July 2018); doi: 10.1117/12.2315618

Event: SPIE Astronomical Telescopes + Instrumentation, 2018, Austin, Texas, United States 


\title{
UV camera conceptual designs for TMT Fiber WFOS
}

\author{
L. Gilles ${ }^{1 \mathrm{a}}$, D. Shafer ${ }^{\mathrm{b}}$, B. Delabre ${ }^{\mathrm{c}}$ \\ ${ }^{a}$ Thirty Meter Telescope International Observatory LLC, 100 W. Walnut St, Pasadena, CA 91124, \\ USA; ${ }^{b}$ David Shafer Optical Design, Fairfield, Connecticut 06824, USA; ${ }^{c}$ European Southern \\ Observatory, Karl Schwarzschild St 2, 85748 Garching, Germany
}

\begin{abstract}
This paper discusses refractive, reflective and catadioptric designs for the Thirty Meter Telescope Fiber Wide Field Optical Spectrograph (WFOS) instrument concept. Custom macros were written to evaluate performance at the detector plane with the grating at the pupil as a function of fiber position in the pseudo-slit and wavelength, and a tolerance analysis has been performed for each design based on best engineering practices to assess performance robustness against opto-mechanical errors. The catadioptric camera appears to provide the best compromise in this regard.
\end{abstract}

Keywords: Wide Field Optical Spectrograph (WFOS) instrument, fiber spectrograph, camera design

\section{INTRODUCTION}

WFOS [1] is a Wide Field Optical Spectrograph, planned to be one of the two first-light instruments for the Thirty Meter Telescope (TMT), together with IRIS, an Infrared Imaging Spectrograph. WFOS is in its conceptual design phase since August 2017. Fiber and slicer concepts are investigated by the WFOS team, and a down-select review is planned towards the end of 2018. This paper describes 4 designs for the ultraviolet (UV) camera of the fiber concept based on first-order spectrograph parameters at the beginning of the conceptual design phase. Two refractive designs are discussed, which are an evolution of previous designs developed by Nick Konidaris and Rebecca Bernstein, a 5-mirror anastigmat (5MA) is discussed, which is an evolution of a freeform design developed by Dave Shafer, and a 3-element catadioptric design is discussed, which is an evolution of a design developed by Bernard Delabre for the MOONS camera. Designs were optimized individually as subsystem components of the whole modular spectrograph instrument. Custom macros were written to evaluate performance at the detector plane for each design with the grating at the pupil as a function of fiber position in the pseudo-slit and wavelength. A tolerance analysis has been performed for each design based on best engineering practices. Iteration with mechanical engineering is required for further refinement of tolerances.

The fiber WFOS straw-man design is inspired from the DESI spectrograph [2][3], which is a 5,000 fiber spectrograph at the Kitt Peak National Observatory (KPNO) and from the MOONS spectrograph [4], which is a 1,000 fiber spectrograph at the Very Large Telescope (VLT). In the fiber WFOS spectrograph concept, the TMT Exit Pupil (XP) would be coupled onto thousands of fibers via microlens arrays (MLAs) positioned via a robotic positioner covering an 8arcmin diameter field of view (FoV) on the TMT Nasmyth focal surface. Light from the TMT XP coupled into fibers at the Nasmyth focal surface would be transported to several (up to nine) rack-mounted replicated modular spectrographs. Each spectrograph would have 3 or 4 arms (see Figure 1), each arm optimized for a specific wavelength band, and the spectrograph would have a total wavelength coverage ranging from $\lambda_{\min }=0.31 \mu \mathrm{m}$ to $\lambda_{\max }=1.0 \mu \mathrm{m}$ and would provide a spectral resolution $R(\lambda)=\lambda / \Delta \lambda=5,000$ across the instrument wavelength band. At the input of each modular spectrograph would be a curved pseudo-slit (object surface) containing hundreds of fibers (circular extended objects) stacked next to each other. The pseudo-slit surface vertex would be at the front focal point of an on-axis spherical collimating mirror whose center of curvature (CC) would coincide with that of the pseudo-slit (both surfaces would be concentric). At the common CC would be a Pupil Plane (PP) located a distance $2 f_{\text {col }}$ from the collimator, where $2 f_{\text {col }}$ denotes the collimator focal length. Because the aperture stop is at the $\mathrm{CC}$ of the spherical collimator, the

\footnotetext{
${ }^{1}$ 1gilles2@email.arizona.edu; phone 1-626-201-4345
}

Ground-based and Airborne Instrumentation for Astronomy VII, edited by Christopher J. Evans, Luc Simard, Hideki Takami, Proc. of SPIE Vol. 10702, 10702AD · C 2018 SPIE · CCC code: 0277-786X/18/\$18 · doi: 10.1117/12.2315618 
spherical mirror does not generate coma or astigmatism but only spherical aberration, which can be compensated by the camera since spherical aberration is field independent. An illustration is provided in Figure 1 (the pseudo-slit is in the page in the $\mathrm{x}$-direction and the camera is modeled as a paraxial lens). All chief rays leave the pseudo-slit (propagating virtually from the common CC) at normal incidence, intercept the collimator at normal incidence, and are retro-reflected along the same ray path onto the pseudo-slit. In this on-axis design, the pseudo-slit thus obscures a few percent of the reflected collimated beam. The collimator re-images the PP to a distance $2 f_{\text {col }}$ from the mirror ( $2 \mathrm{f}$ imaging), where a tilted grating is placed to disperse light from each fiber in the pseudo-slit into a spectrum (Y direction on the detector).

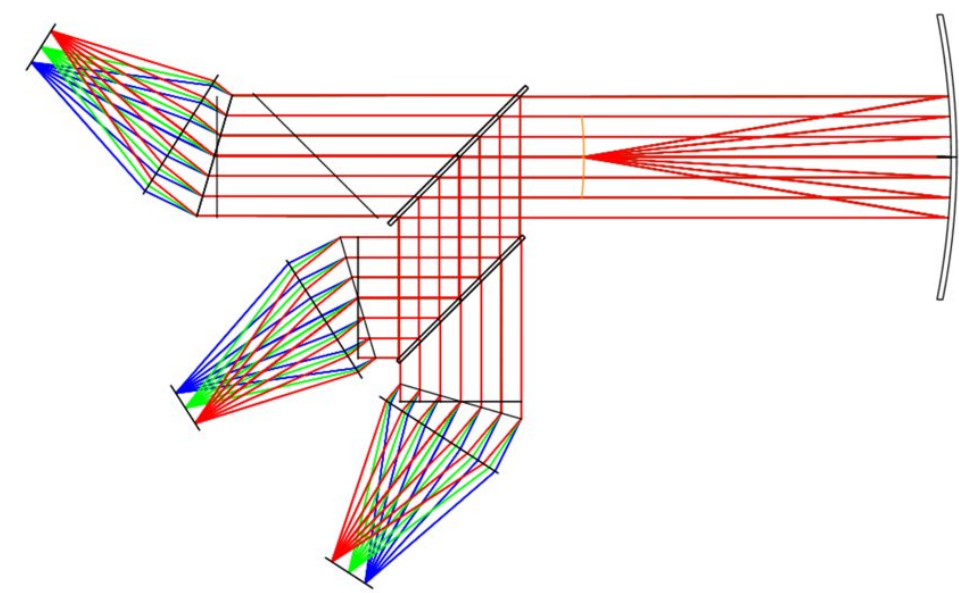

Figure 1: Layout of a modular 3-channel fiber spectrograph.

In this paper, we are concerned with the most challenging ultraviolet (UV) arm of such modular spectrograph, with waveband

$$
\lambda_{1}=0.31 \mu \mathrm{m}, \quad \lambda_{2}=0.45 \mu \mathrm{m}, \quad \lambda_{0}=0.38 \mu \mathrm{m}
$$

where $\lambda_{0}$ denotes the primary wavelength at which focal lengths are specified. For maximum grating efficiency versus wavelength (minimum angular deviation), the spectrograph is designed in Littrow configuration [5][6]. In this configuration, the diffraction angle of the chief ray of the first diffraction order at the primary wavelength $\beta\left(\lambda_{0}\right)>0$, is equal to the tilt angle of the grating, $\alpha>0$, as illustrated in Figure 2. The angle between the collimator axis and the camera axis is $\psi=\alpha+\beta\left(\lambda_{0}\right)$. In this paper, a grating period equal to $v=1.475 \mathrm{ln} / \mu \mathrm{m}$ is assumed. The diffraction angle, $\beta(\lambda)$, is related to the grating tilt angle by the grating equation:

$$
\beta(\lambda)=\sin ^{-1}\left[m_{d} v \lambda-\sin (\alpha)\right],
$$

where $m_{d}$ denotes the diffraction order and $v$ the grating period. To first-order, the chief ray diffraction angle $\beta(\lambda)$ in (1.2) may be linearized with respect to the grating tilt angle $\alpha$ and wavelength $\lambda$ :

$$
\beta(\lambda)[\mathrm{deg}] \sim \frac{m_{d} v \lambda}{\pi} 180[\mathrm{deg}]-\alpha[\mathrm{deg}]
$$

The Littrow configuration is found by setting $\beta\left(\lambda_{0}\right)=\alpha$ in (1.2) and solving for $\alpha$ :

$$
\begin{aligned}
& \beta\left(\lambda_{0}\right)-\alpha=0 \Rightarrow \alpha=\beta\left(\lambda_{0}\right)=\sin ^{-1}\left[m_{d} v \lambda_{0}-\sin (\alpha)\right] \\
& \Rightarrow \sin (\alpha)=m_{d} v \lambda_{0}-\sin (\alpha) \Rightarrow \sin (\alpha)=\frac{m_{d} v \lambda_{0}}{2} \\
& \Rightarrow \alpha=\sin ^{-1}\left[\frac{m_{d} v \lambda_{0}}{2}\right]=16.275126^{\circ}
\end{aligned}
$$


Plots of chief ray diffraction angle versus wavelength $\beta(\lambda)$ and of its change versus wavelength $d \beta / d \lambda$ are provided in Figure 3. The camera field of view (FoV) along the dispersion direction (Y-direction) is given by

$$
\mathrm{FoV}_{y}=\beta\left(\lambda_{2}\right)-\beta\left(\lambda_{1}\right)=12.4^{\circ}
$$

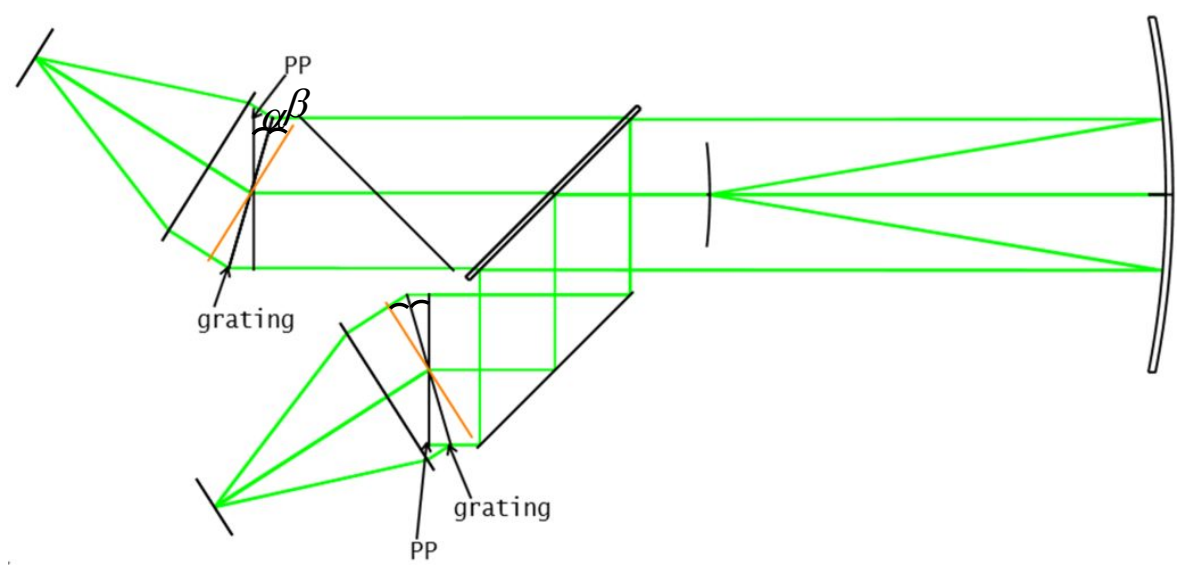

Figure 2: Littrow spectrograph configuration for maximum diffraction grating efficiency.
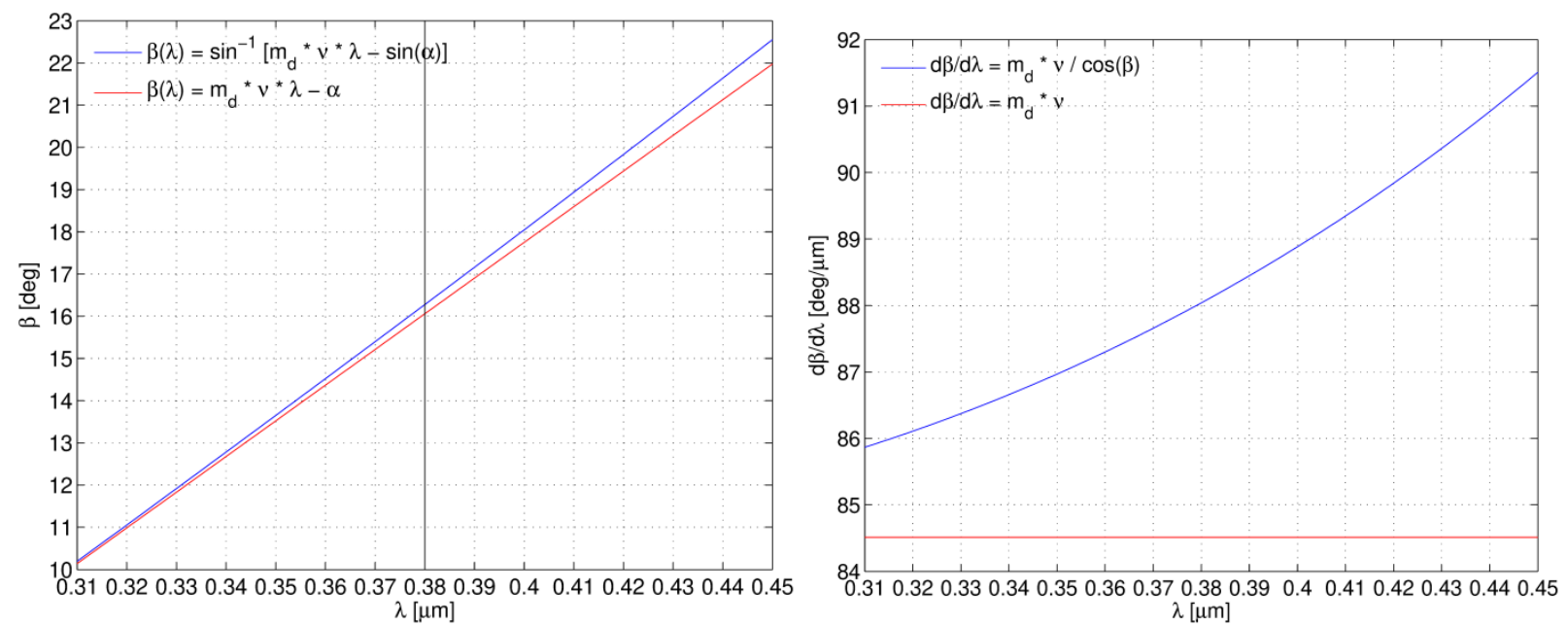

Figure 3: Left: chief ray diffraction angle versus wavelength. Right: change in chief ray diffraction angle with wavelength.

A driving first-order property is the spectrograph paraxial transverse magnification $m$ whose value was chosen based on the DESI spectrograph design. Namely, the spectrograph would image a $100 \mu \mathrm{m}$ diameter fiber object onto a first-order circular image of diameter $45 \mu \mathrm{m}$ (i.e. three $15 \mu \mathrm{m}$ pixels), i.e.

$$
m\left(\lambda_{0}\right)=\frac{h^{\prime}\left(\lambda_{0}\right)}{h}=-\frac{f_{\text {cam }}\left(\lambda_{0}\right)}{f_{\text {col }}}=-0.45
$$

where $h=50 \mu \mathrm{m}$ denotes object height, $h^{\prime}=-22.5 \mu \mathrm{m}$ image height, and $f_{\text {cam }}$ is the camera focal. The first-order image diameter $2\left|h^{\prime}\right|$ is an important first-order parameter; it places a limit on spectral resolution: resolving two wavelengths separated by a spectral resolution element $\Delta \lambda$ requires the two images to be separated along the dispersion direction by a spatial resolution element $\Delta x$ at least as large as their diameter:

$$
\Delta x\left(\lambda_{0}\right) \geq 2\left|h^{\prime}\left(\lambda_{0}\right)\right|
$$


The ultraviolet band places serious constraints on the available glasses for the camera. It appears that only Calcium Fluoride (CaF2) and Fused Silica ( $\mathrm{SiO} 2)$ glasses have a transmittance greater than $99.8 \%$ at these wavelengths for $10 \mathrm{~mm}$ thick samples, see for instance [21]. In this paper, we are considering a $6 \mathrm{~K}$ x $6 \mathrm{~K}$ sensor with $15 \mu \mathrm{m}$ pixels, providing a square $L^{\prime} \times L^{\prime}$ image size with $L^{\prime}=90 \mathrm{~mm}$, e.g. a $3 \times 3$ mosaic of the e2v CCD230-42 [21]. Given the value for the spectrograph transverse magnification, one finds that a $L=L^{\prime} /\left|m\left(\lambda_{0}\right)\right|=90 / 0.45=200 \mathrm{~mm}$ pseudo-slit fits the image size.

\section{BLUE CHANNEL CAMERA REQUIREMENTS}

\subsection{First-order properties}

Driving design parameters for the blue camera flow down from the following top-level spectrograph parameters:

1. The camera focal length $f_{\text {cam }}$ is defined such that its product with the tangent of the angular resolution element $\Delta \beta$ (angle between 2 resolvable wavelengths) is equal to the spatial resolution element $\Delta x$ along the dispersion direction as given by (1.7) (linear distance on the detector between 2 resolvable wavelengths, i.e. spectral FWHM):

$$
f_{\text {cam }}\left(\lambda_{0}\right) \tan \left(\Delta \beta\left(\lambda_{0}\right)\right)=\Delta x\left(\lambda_{0}\right)
$$

The angular resolution element $\Delta \beta$ is obtained by differentiating the grating equation (1.2) with respect to wavelength, which yields:

$$
\Delta \beta\left(\lambda_{0}\right)=\frac{m_{d} v \lambda_{0}}{R\left(\lambda_{0}\right) \cdot \cos \left(\beta\left(\lambda_{0}\right)\right)}
$$

In the limit of small $\Delta \beta$, we have $\tan (\Delta \beta) \sim \Delta \beta$, and (1.8) can be rewritten:

$$
f_{\text {cam }}\left(\lambda_{0}\right)=\Delta x\left(\lambda_{0}\right) \cdot R\left(\lambda_{0}\right) \cdot \frac{\cos \left(\beta\left(\lambda_{0}\right)\right)}{m_{d} v \lambda_{0}}
$$

For the Littrow configuration, (1.10) simplifies to:

$$
f_{\text {cam }}\left(\lambda_{0}\right)=\Delta x\left(\lambda_{0}\right) \cdot R\left(\lambda_{0}\right) \cdot \frac{1}{2 \tan (\alpha)}=392 \mathrm{~mm}
$$

The fiber WFOS instrument is required to have a spectral resolution equal to $R\left(\lambda_{0}\right)=\lambda_{0} / \Delta \lambda=5,000$ (i.e. $0.076 \mathrm{~nm}$ wavelength resolution around $380 \mathrm{~nm}$ ), which for the choice of grating angle and spectrograph magnification leads to a $392 \mathrm{~mm}$ camera focal length at the primary wavelength and $\Delta \beta\left(\lambda_{0}\right) \sim 24 \operatorname{arcsec}$.

2. From (1.6), the collimator focal length must be longer than the camera focal length by a factor $1 /\left|m\left(\lambda_{0}\right)\right|$, leading to a collimator focal length of $872 \mathrm{~mm}$ :

$$
f_{\text {col }}=\frac{f_{\text {cam }}\left(\lambda_{0}\right)}{\left|m\left(\lambda_{0}\right)\right|}=872 \mathrm{~mm}
$$

3. The collimator should be faster than the MLA to account for focal ratio degradation (FRD) introduced by the fiber [9]. Based on the DESI design, a microlens (ML) focal ratio equal to 3.2 is adopted, and a collimator focal ratio of 3.0:

$$
f_{\# \mathrm{ML}}\left(\lambda_{0}\right)=3.2, \quad f_{\# \mathrm{col}}=3.0
$$

As a side note, the ML focal length can be calculated from the imaging equation since the object (TMT XP) distance from the TMT FP and the ML magnification are known. The TMT XP is located at a distance $z_{\mathrm{XP}}=-46,386.61 \mathrm{~mm}$ from the TMT FP and its semi-diameter is equal to $h_{\mathrm{XP}}=1,546.2 \mathrm{~mm}$. The required ML magnification to image a point 
on the TMT XP perimeter onto a first-order image of height equal to $h_{\mathrm{XP}}^{\prime}=-0.05 \mathrm{~mm}$ is thus $m_{\mathrm{ML}}=h_{\mathrm{XP}}^{\prime} / h_{\mathrm{XP}}=-0.1 / 3,092.4=-(3.2337) \cdot 10^{-5}$. Since image and object locations are related by magnification $m_{\mathrm{ML}}=\left(z_{\mathrm{XP}}^{\prime} / n^{\prime}\right) /\left(z_{\mathrm{XP}} / n\right)$, we have $z_{\mathrm{XP}}^{\prime}=1.5 \mathrm{~mm}$, and therefore the $\mathrm{ML}$ optical power is given by $\phi_{\mathrm{ML}}=1 / f_{\mathrm{ML}}=\Delta\left\{n / z_{\mathrm{XP}}\right\}=n^{\prime} / z^{\prime}{ }_{\mathrm{XP}}-n / z_{\mathrm{XP}}=0.6668 / \mathrm{mm}$, leading to $f_{\mathrm{ML}}=1.5 \mathrm{~mm}$. Given the focal ratio value given in eq.(1.13), the ML diameter is equal to $d_{\mathrm{ML}}=f_{\mathrm{ML}} / f_{\# \mathrm{ML}}=0.4688 \mathrm{~mm}$, which corresponds to an angular subtense equal to $\theta_{\mathrm{ML}}=d_{\mathrm{ML}} / f_{\mathrm{ML}}=0.215 \operatorname{arcsec}$. Combining (1.12) and (1.13) yields the camera Entrance Pupil (EP) size:

$$
D_{E P}=f_{\text {col }} / f_{\text {\#col }}=291 \mathrm{~mm}
$$

Combining (1.12) and (1.13) yields the camera focal ratio:

$$
f_{\text {\# } \mathrm{cam}}\left(\lambda_{0}\right)=f_{\text {cam }}\left(\lambda_{0}\right) / D_{E P}=1.35
$$

The camera field of view along the spatial (pseudo-slit angular subtense) direction can be calculated as follows. For the spatial direction, for a flat object surface, we have $f_{\text {col }} \tan \left(\mathrm{FoV}_{x} / 2\right)=L / 2=100 \mathrm{~mm}$, hence FoV ${ }_{x} / 2=6.54^{\circ}$. Taking into account the curvature of the object surface, a value equal to $\mathrm{FoV}_{x} / 2=6.59^{\circ}$ is obtained, hence the field of view along the spatial direction is given by:

$$
\mathrm{FoV}_{x}=13.2^{\circ}
$$

The spatial spread in the dispersion direction for the on-axis fiber is given by:

$$
l_{y}=2 f_{\text {cam }}\left(\lambda_{0}\right) \tan \left(\mathrm{FoV}_{y} / 2\right)=84.93 \mathrm{~mm}
$$

where $\mathrm{FoV}_{y}$ is given in (1.5). A real ray-trace from the curved object surface returns a diagonal field of view equal to:

$$
\mathrm{FoV}=18.6^{\circ}
$$

It is clear that the camera speed, large FoV and wavelength range pose significant design challenges.

\subsection{Performance requirements}

The spectrograph performance metric is encircled energy (EE). The requirement is not well defined at this stage of the CODP, but an end-to-end value for the spectrograph of $90 \% \mathrm{EE}$ inside the first-order image of a fiber diameter $\left(h^{\prime}=-22.5 \mu \mathrm{m}, h=50 \mu \mathrm{m}\right)$ over the field and wavelength range would be excellent. This performance requirement is extremely ambitious, and corresponds to near-diffraction limited performance (below half wave RMS wavefront error (WFE)). In angular space, the $h=50 \mu \mathrm{m}$ circular fiber radius subtends an angle $\theta_{\mathrm{obj}} \operatorname{satisfying} f_{\text {col }} \tan \left(\theta_{\mathrm{obj}}\right)=h$. The angular subtense of the fiber diameter (object) is then:

$$
2 \theta_{\text {obj }}=23.65 \operatorname{arcsec}=(6.57) \cdot 10^{-3} \mathrm{deg}
$$

This value matches $\Delta \beta\left(\lambda_{0}\right)$ and is used as object angular size to compute the Extended Source EE.

\subsection{Design constraints}

The following constraints are imposed on the design of the camera:

1. The stop should be external at distance of at least $85 \mathrm{~mm}$ in front of the first element of the camera. This distance is called "pupil relief" (PR).

2. $0 \%$ vignetting.

3. The back focal distance (BFD) should be at least $8 \mathrm{~mm}$ to accommodate the CCD window. 


\section{REFRACTIVE CAMERA DESIGNS}

\subsection{Design progression}

An 11-element spherical lens (denoted "11Sph-0Asph") developed by Nick Konidaris [10], and an aspheric lens developed by Rebecca Bernstein using 3 spherical lenses plus 6 aspheric lenses (denoted "3Sph-6Asph") [11] served as our starting point. The aspheric lenses in Rebecca's design have the front surface of lens 1, 3, 5, 7, and 8 modeled as the sum of $4^{\text {th }}, 6^{\text {th }}$, and $8^{\text {th }}$-order radially-symmetric asphericities, i.e.

$$
\begin{aligned}
& \operatorname{sag}(\rho)=\operatorname{sag}_{\text {conicoid }}(\rho)+a_{4} \rho^{4}+a_{6} \rho^{6}+a_{8} \rho^{8}, \\
& \rho=\sqrt{x^{2}+y^{2}}, \quad \operatorname{sag}_{\text {conicoid }}(\rho, K)=\frac{c \rho^{2}}{1+\sqrt{1-(1+K) c^{2} \rho^{2}}}
\end{aligned}
$$

where $c=1 / R$ denotes curvature, $R$ radius of curvature and $\rho$ transverse radial distance. In both design, fused silica ( $\mathrm{SiO} 2)$ elements are negative elements (negative focal lengths), calcium fluoride (CaF2) elements are positive elements (positive focal lengths).

- Step1. We started by updating the waveband to the values given in (1.1), scaling both designs to the desired focal length given in (1.11), setting $D_{\mathrm{EP}}=191 \mathrm{~mm}$ which yields $f_{\text {\#,cam }}=2$, and defining a circular field of view of diameter given in (1.18). The grating is not included in the optical model at this stage. All field points within the circular field of view are simulated at all wavelengths, and axial symmetry is assumed. Those two simplifying assumptions will be dropped when analyzing performance of the camera with the grating included at the pupil plane and all fibers in the pseudo-slit simulated. As a starting point, to speed up ray tracing during the optimization procedure, waveband and FoV were sampled at only 3 points (min, max, mid-point). The following constraints were imposed on the design in the merit function (MF):

$\circ$ thickness of each lens element between $15 \mathrm{~mm}$ (center or edge) and $120 \mathrm{~mm}$ (center),

○ spacing between elements between $0.5 \mathrm{~mm}$ (center or edge) and $100 \mathrm{~mm}$ (center),

○ focal length $392 \mathrm{~mm}$ at the primary wavelength,

- BFD at least $8 \mathrm{~mm}$.

Lateral color (LAC, i.e. chromatic change of transverse magnification) was ignored. Pupil integration was performed using Gaussian quadrature (GQ) with 10 rings and 10 arms. This integration method is significantly faster and requires significantly less computer memory than a fine rectangular grid, which typically requires very high sampling to reach percent level fluctuations in MF value as sampling changes. Levenberg-Marquardt damped least squares (DLS) optimization was performed on both designs (convergence is slow and reaching a local minimum takes several hours on an 8-core laptop computer).

- Step2. The entrance pupil diameter (EPD) was increased to $D_{\mathrm{EP}}=217 \mathrm{~mm}\left(f_{\#, \text { cam }}=1.8\right)$ and both designs were reoptimized, paying close attention to constraints violations.

- Step3. The EPD was increased to $D_{\mathrm{EP}}=245 \mathrm{~mm}\left(f_{\#, \text {,am }}=1.6\right)$ and both designs were re-optimized.

- Step4. The EPD was increased to $D_{\mathrm{EP}}=270 \mathrm{~mm}\left(f_{\#, \text {,am }}=1.45\right)$ and both designs were re-optimized.

- This step required lens center thicknesses of up to $180 \mathrm{~mm}$.

- Step5. Both designs were re-optimized for the target EPD i.e. $D_{\mathrm{EP}}=291 \mathrm{~mm}\left(f_{\# \text {,cam }}=1.35\right)$. The total mass of both designs produced after optimization is $460 \mathrm{~kg}$ for the 11 -element design and $205 \mathrm{~kg}$ for the 9 -element design.

- Step6. The following modifications were made in the MF:

- The glass minimum center thickness was increased to $25 \mathrm{~mm}$ and edge thickness to $20 \mathrm{~mm}$ respectively,

$\circ \quad$ The minimum spacing between elements was increased to $1 \mathrm{~mm}$. 
- A 55deg constraint was imposed on the maximum ray angle of incidence and exitance at each surface to reduce near total internal reflection (TIR) observed at Step5.

- $\quad$ Step7.

- A maximum total mass constraint of $370 \mathrm{~kg}$ for 11 -element design and of $265 \mathrm{~kg}$ for the 9-element design was added in the MF.

○ The spacing between elements was allowed to grow up to $200 \mathrm{~mm}$.

- A 10\% margin was imposed on the clear semi-diameters of all elements.

○ Pupil relief was allowed to vary between $85-100 \mathrm{~mm}$ for the 11-element design.

- The constraint on the maximum ray angle of incidence and exitance was increased to 65deg for the 11element design.

- $\quad$ Step8.

○ The last element (aspheric field lens) of the 9-element design was removed (the resulting design will be denoted "3Sph-5Asph").

- The MF was updated for finer field and wavelength sampling: 11 Y-fields, 11 wavelengths, and both systems were re-optimized.

- Step9.

○ The grating was introduced at the pupil, $11 \mathrm{X}$-fields and 11 wavelengths were simulated, the MF was updated and both systems were re-optimized.

\subsection{Performance Result}

\subsubsection{Spherical lens with grating and fibers in pseudo-slit}

\subsubsection{Nominal performance}

In this Section, an in-depth performance analysis of the lens including the grating and fibers in the pseudo-slit is discussed. The simulated $11 \mathrm{x}$-field points sampling the spectrograph pseudo-slit have coordinates given by $\theta_{x}=\left\{0^{\circ}, \pm 1.32^{\circ}, \pm 2.64^{\circ}, \pm 3.96^{\circ}, \pm 5.28^{\circ}, \pm 6.6^{\circ}\right\}$. The lens layout and the optical prescription are shown in Figure 4 and Figure 5 respectively. All radii and thicknesses are specified with only 2 significant digits (10 microns accuracy) and the optimization criterion was RMS WFE with respect to centroid over all $11 \mathrm{x}$-fields and 11 wavelengths, and pupil integration was performed using GQ with 10 rings and 10 arms. Uniform field and wavelength weights were used to build the merit function. As-designed performance results are shown in Figure 6 (performance is symmetric with respect to $\mathrm{x}$-fields since the as-designed lens system is axially symmetric). Sensitivity to BFD and image plane tilt is illustrated in Figure 7. The mean extended source EE drops by $10 \%$ for a $\pm 40 \mu \mathrm{m}$ BFD error about the nominal value, or for a tilt error equal to $\pm 0.1 \mathrm{deg}$.

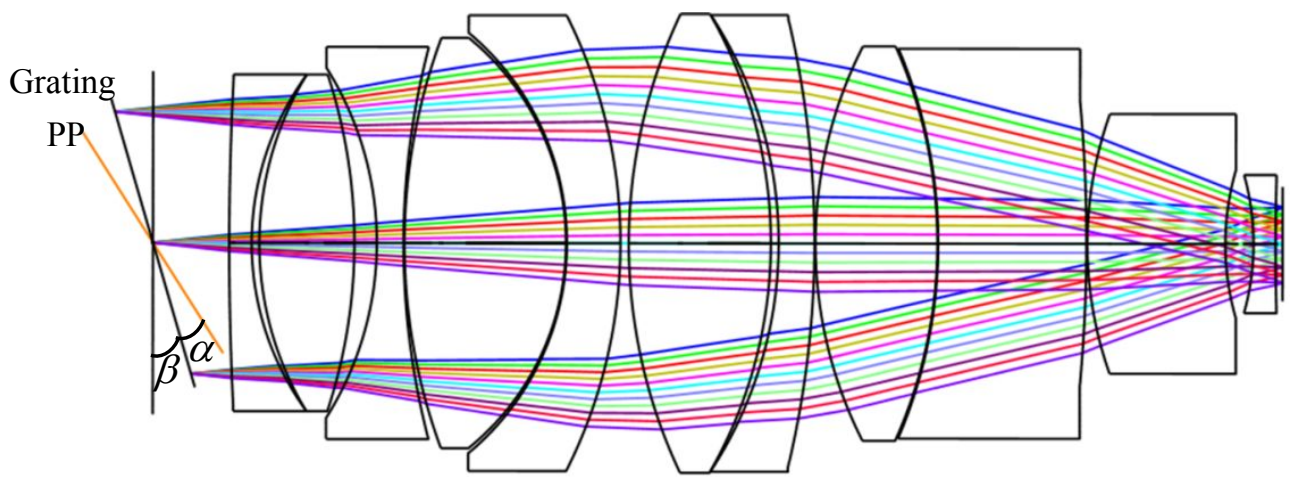

Figure 4: Optical layout and on-axis field Y-fan for the $11 \mathrm{Sph}-0 \mathrm{Asph}$ design including the grating. The global coordinate reference surface is the front surface of the first lens. Rays are colored by wavelength. 


\begin{tabular}{|c|c|c|c|c|c|c|c|c|c|c|c|c|c|}
\hline 4 & & Surt:Type & Comment & Radius & Thickness & Material & Semi-Diameter & Mech Semi-Dia & Conic & Decenter X & Decenter Y & Tilt About X & Tilt About Y \\
\hline 0 & ОВЈЕСТ & Standard $\mathbf{v}$ & & Infinity & Infinity & & Infinity & Infinity & 0.000000 & & & & \\
\hline 1 & STOP & Standard $\boldsymbol{v}$ & pupil & Infinity & 0.000000 & & 145.500000 & 145.500000 & 0.000000 & & & & \\
\hline 2 & & Coordinate Break - & & & 0.000000 & & 0.000000 & - & & 0.000000 & 0.000000 & 16.280000 & 0.000000 \\
\hline 3 & & Diffraction Grating & grating & Infinity & 0.000000 & & 167.385276 & - & 0.000000 & 1.475000 & -1.000000 & & \\
\hline 4 & & Coordinate Break - & pupil relief & & $84.974008 \mathrm{~V}$ & & 0.000000 & - & & 0.000000 & 0.000000 & 16.270252 & 0.000000 \\
\hline 5 & & Standard $=$ & lens 1 & 3580.150000 & 25.000000 & F_STUCA & 181.042576 & 187.106134 & 0.000000 & & & & \\
\hline 6 & & Standard $\mathbf{V}$ & & 316.230000 & 8.500000 & & 187.106134 & 187.106134 & 0.000000 & & & & \\
\hline 7 & & Standard - & lens 2 & 359.140000 & 105.950000 & CAF2 & 188.742634 & 194.108742 & 0.000000 & & & & \\
\hline 8 & & Standard $\mathbf{v}$ & & -598.020000 & 24.960000 & & 194.108742 & 194.108742 & 0.000000 & & & & \\
\hline 9 & & Standard $=$ & lens 3 & -363.880000 & 29.750000 & F_SILCA & 194.494319 & 217.890790 & 0.000000 & & & & \\
\hline 10 & & Standard $\boldsymbol{v}$ & & 863.980000 & 1.000000 & & 217.890790 & 217.890790 & 0.000000 & & & & \\
\hline 11 & & Standard . & lens 4 & 656.730000 & 179.940000 & CAF2 & 223.446955 & 235.081724 & 0.000000 & & & & \\
\hline 12 & & Standard $\mathbf{v}$ & & -293.890000 & 1.000000 & & 235.081724 & 235.081724 & 0.000000 & & & & \\
\hline 13 & & Standard $\mathbf{v}$ & lens 5 & -303.680000 & 59.650000 & F.SIUCA & 233.591268 & 253.197535 & 0.000000 & & & & \\
\hline 14 & & Standard $\boldsymbol{V}$ & & -562.210000 & 9.290000 & & 253.197535 & 253.197535 & 0.000000 & & & & \\
\hline 15 & & Standard $\mathbf{V}$ & lens 6 & 595.600000 & 158.250000 & CAF2 & 260.428708 & 260.428708 & 0.000000 & & & & \\
\hline 16 & & Standard $\mathbf{v}$ & & -510.980000 & 8.680000 & & 255.006529 & 260.428708 & 0.000000 & & & & \\
\hline 17 & & Standard $\mathbf{v}$ & lens 7 & -462.250000 & 39.980000 & F_SLUCA & 253.730901 & 253.730901 & 0.000000 & & & & \\
\hline 18 & & Standard $\boldsymbol{V}$ & & -1069.010000 & 1.000000 & & 249.354996 & 253.730901 & 0.000000 & & & & \\
\hline 19 & & Standard $\mathbf{v}$ & lens 8 & 482.820000 & 135.860000 & CAF2 & 233.906384 & 233.906384 & 0.000000 & & & & \\
\hline 20 & & Standard $\boldsymbol{V}$ & & -535.580000 & 0.870000 & & 219.204332 & 233.906384 & 0.000000 & & & & \\
\hline 21 & & Standard - & lens 9 & -548.970000 & 165.110000 & F_SLUCA & 216.636742 & 216.636742 & 0.000000 & & & & \\
\hline 22 & & Standard $\mathbf{v}$ & & -1733.130000 & 0.990000 & & 158.702633 & 216.636742 & 0.000000 & & & & \\
\hline 23 & & Standard - & lens 10 & 430.110000 & 153.730000 & F_StuCA & 144.283978 & 144.283978 & 0.000000 & & & & \\
\hline 24 & & Standard $\boldsymbol{V}$ & & 295.530000 & 31.380000 & & 82.428781 & 144.283978 & 0.000000 & & & & \\
\hline 25 & & Standard $\mathbf{v}$ & lens 11 & -283.410000 & 24.580000 & F_SILCA & 76.822599 & 76.822599 & 0.000000 & & & & \\
\hline 26 & & Standard $\boldsymbol{V}$ & & 2632.360000 & $7.840000 \mathrm{~V}$ & & 71.388145 & 76.822599 & 0.000000 & & & & \\
\hline 27 & & Coordinate Break - & & & 0.000000 & & 0.000000 & . & & 0.000000 & 0.000000 & $0.000000 \mathrm{v}$ & $0.000000 \mathrm{~V}$ \\
\hline 28 & IMAGE & Standard $\boldsymbol{V}$ & & Infinity & . & & 63.566902 & 63.566902 & 0.000000 & & & & \\
\hline
\end{tabular}

Figure 5: Optical prescription for the 11Sph-0Asph design including the grating.
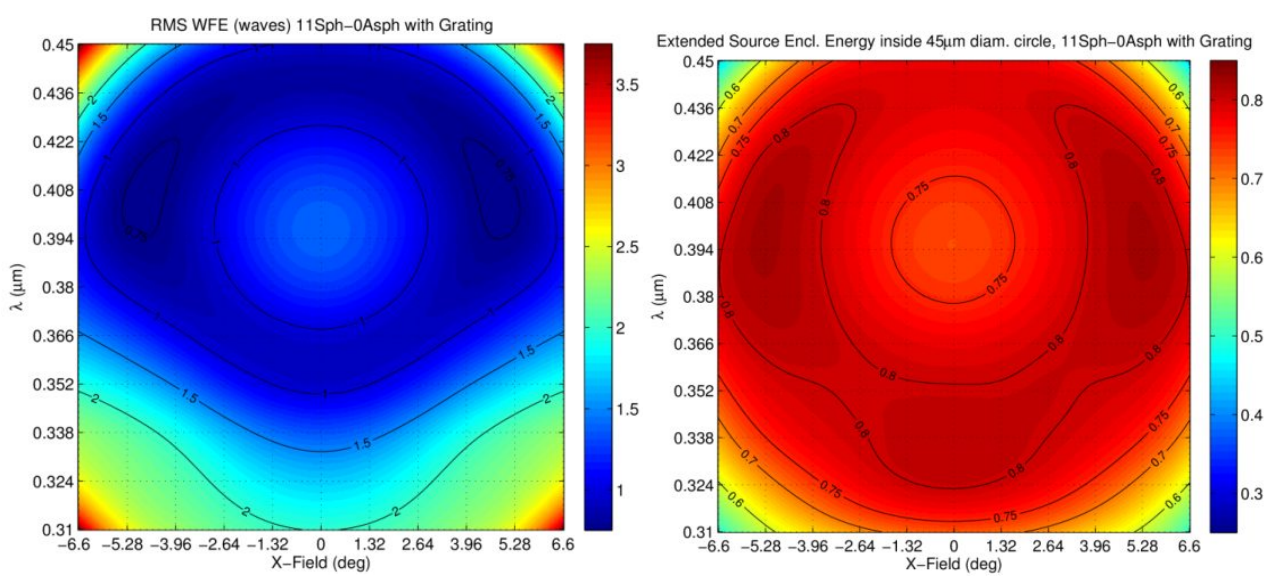

Figure 6: RMS WFE and extended source EE within $45 \mu \mathrm{m}$ diameter versus field and wavelength for the 11-element spherical lens with grating. The RMS WFE map is plotted with color bar on a scale ranging from 0.75 to 3.75 waves, and the EE map from 0.25 to 0.85 .
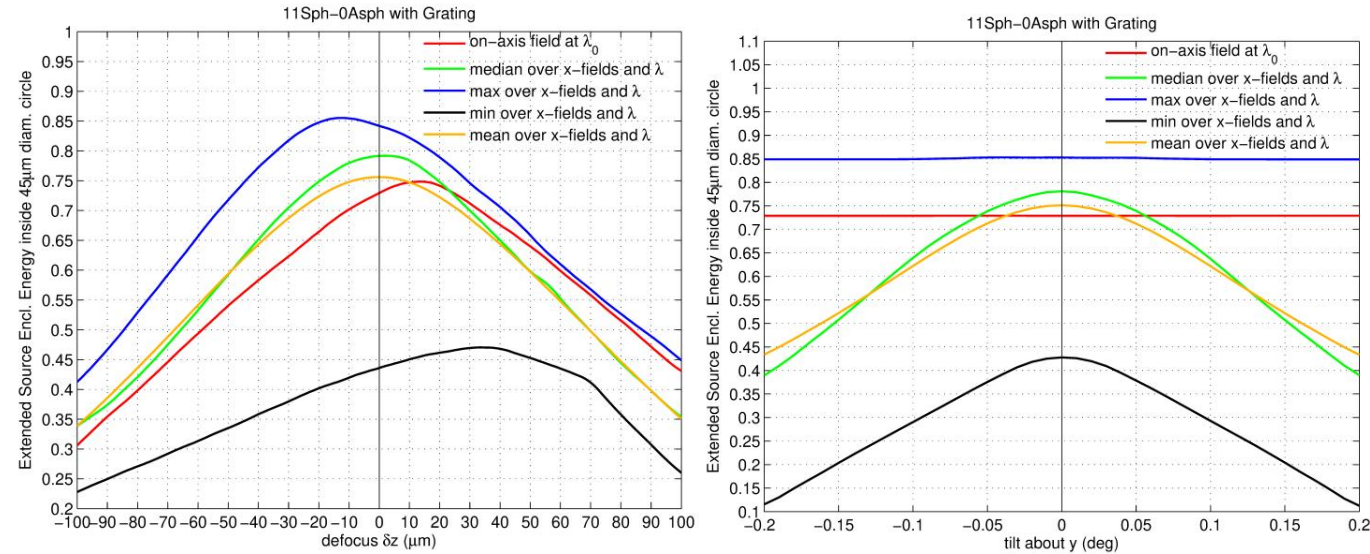

Figure 7: Left: extended source EE within $45 \mu \mathrm{m}$ diameter versus defocus from nominal image plane position. Right: extended source encircled energy sensitivity to detector plane tilt. 


\subsubsection{Tolerance analysis}

An in-depth tolerance analysis was performed, considering all $\mathrm{x}$-fields and wavelengths. Each Monte Carlo trial was optimized for RMS WFE over all $\mathrm{x}$-fields and wavelengths. A custom external macro was used to post-process the saved Monte Carlo runs and compute the extended source EE for each field point, wavelength and simulation trial. Since this process is computationally intensive, EE was queried only over an $11 \mathrm{x} 11$ grid of points $(11 \mathrm{x}$-fields and 11 wavelengths). The tolerances listed in Table 1 were imposed on the lens based on Optimax manufacturing tolerance chart [12]. Monte Carlo tolerance analysis is critical to understand product cost versus performance [13].

\begin{tabular}{|l|l|l|}
\hline Item & Value & Comment \\
\hline Radius of curvature & $\pm 0.1 \%$ or $\pm 0.05 \%$ & from sensitivity analysis \\
\hline Thickness (glass and air) & $\pm 100 \mu \mathrm{m}$ & $\begin{array}{l}\text { twice Optimax high-precision thickness } \\
\text { tolerance as an estimate for linear distance } \\
\text { tolerance, pending mechanical review }\end{array}$ \\
\hline X and Y element decenter & $\pm 50 \mu \mathrm{m}$ & Optimax high precision \\
\hline Surface irregularity & \pm 0.2 fringes & Optimax high precision \\
\hline $\begin{array}{l}\text { Surface X and Y tilt, i.e. } \\
\text { (wedge) }\end{array}$ & $\pm 2.5 \mu \mathrm{m}$ & Optimax wedge prism high precision \\
\hline \begin{tabular}{l} 
Element X and Y tilt \\
\hline Index
\end{tabular} & $\pm 0.0083 \mathrm{deg}( \pm 0.5$ arcmin $)$ & $\pm 5 \cdot 10^{-4}$ \\
\hline Abbe number & $\pm 0.8 \%$ & \multicolumn{2}{|c|}{} \\
\hline
\end{tabular}

Four active compensation strategies were studied in the 1,000 Monte Carlo simulation trials with uniform optomechanical error statistics:

- Active BFD adjustment (constrained between $6 \mathrm{~mm}$ and $13 \mathrm{~mm}$ )

- Active Pupil Relief adjustment (constrained between $83 \mathrm{~mm}$ and $90 \mathrm{~mm}$ )

- Active detector plane X-and Y-tilt adjustment (constrained between -2deg and +5deg)

Figure 8 plots performance versus success rate (defined as the probability over Monte Carlo trials to meet or exceed a given performance level). We find that the median extended source encircled energy over $\mathrm{x}$-fields and wavelengths is slightly worse than on-axis encircled energy at the primary wavelength.

- For a 98\% success rate, $35 \%$ median extended source encircled energy over $\mathrm{x}$-fields and wavelengths can be expected.

- For a $90 \%$ success rate, $43 \%$ median extended source encircled energy over $\mathrm{x}$-fields and wavelengths can be expected.

- For a $70 \%$ success rate, $51 \%$ median extended source encircled energy over $\mathrm{x}$-fields and wavelengths can be expected.

Compensator statistics from the 1,000 Monte Carlo simulations illustrate that the mean pupil relied is very close to $85 \mathrm{~mm}$, mean BFD very close to $8 \mathrm{~mm}$ and mean image tilt about the $\mathrm{x}$-axis close to 6milli-deg (0.36arcmin). 


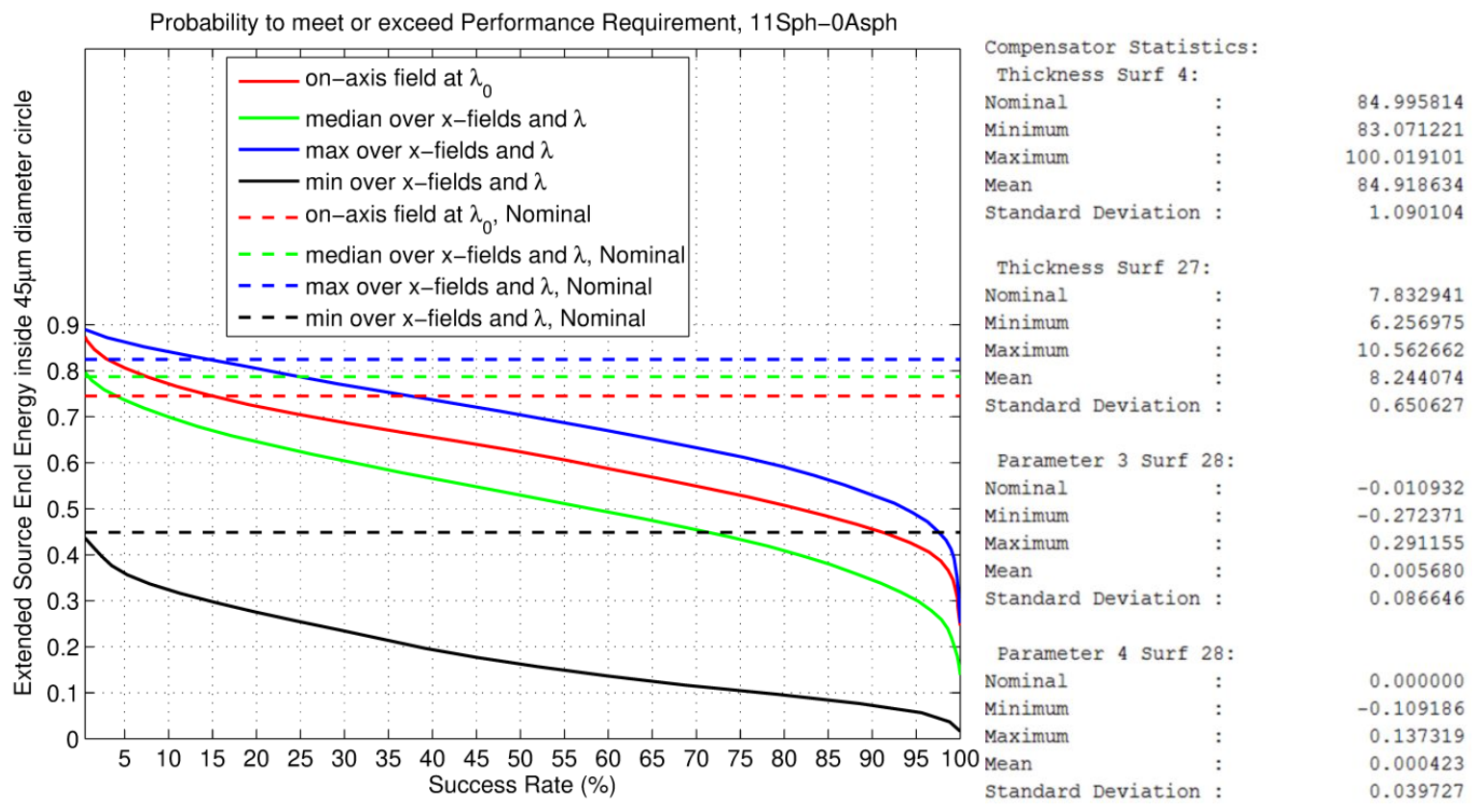

Figure 8: Monte Carlo tolerance results of the spherical lens with grating. 1,000 Monte Carlo trials were performed with uniform opto-mechanical error statistics. Success rate is defined as the probability over simulation trials to meet or exceed a given performance level. Right: compensator statistics for the $11 \mathrm{Sph}-0 \mathrm{Asph}$ design including the grating. The first group is pupil relief, the second is BFD and last two are image plane tilt about the $\mathrm{x}$ - and $\mathrm{y}$-axis respectively.

\subsubsection{Aspheric lens with grating and fibers in pseudo-slit}

\subsubsection{Nominal performance}

In this Section, performance of the aspheric lens with the grating and all fibers in the pseudo-slit is discussed. The lens layout is show in Figure 9, the optical prescription in Figure 10 and performance results in Figure 11 and Figure 12. The merit function was built with the on-axis and edge $\mathrm{x}$-field points having twice the weight of the other $\mathrm{x}$-fields, and similarly the primary and edge wavelengths having twice the weight of the other wavelengths. Such a weighting provides more uniform performance across fields and wavelengths.

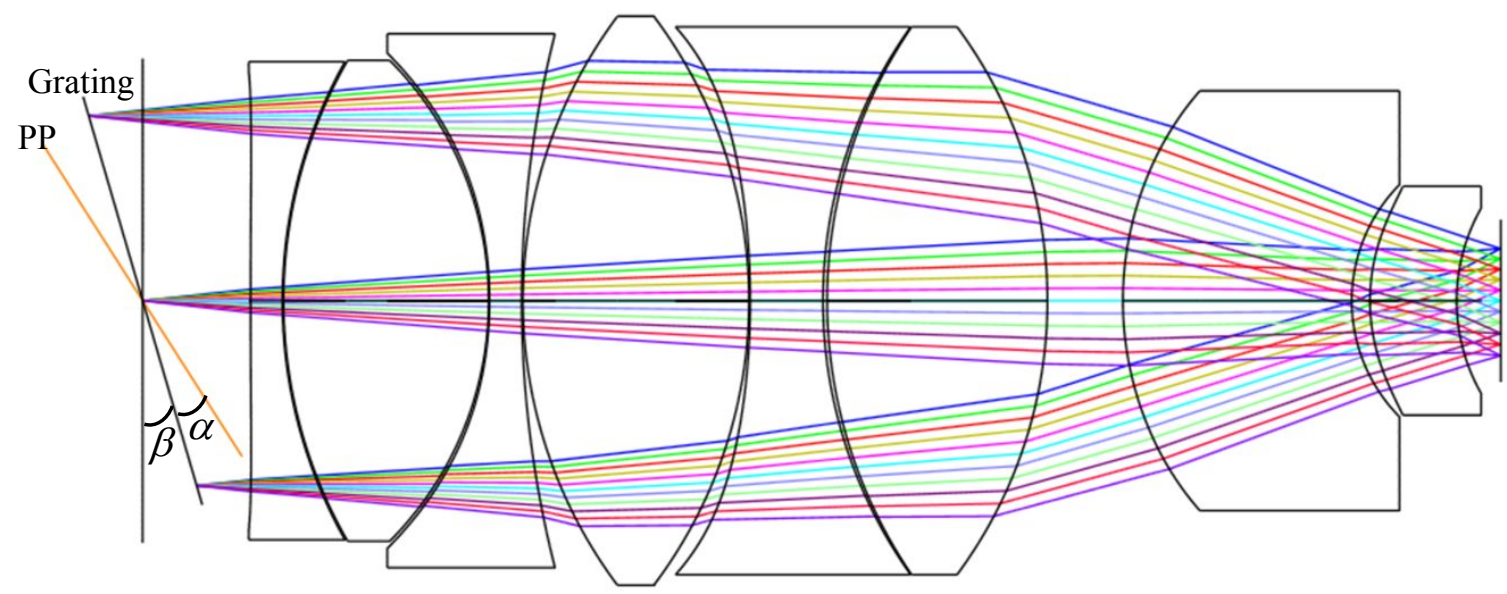

Figure 9: Optical layout and on-axis field Y-fan for the 3Sph-5Asph design including the grating. The global coordinate reference surface is the front surface of the first lens. Rays are colored by wavelength. 


\begin{tabular}{|c|c|c|c|c|c|c|c|c|c|c|c|c|c|}
\hline 4 & & Surf:Type & Comment & Radius & Thickness & Material & Semi-Diameter & Mech Semi-Dia & Conic & Decenter X & Decenter Y & Tilt About X & Tilt About Y \\
\hline 0 & OBJECT & Standard $\mathbf{v}$ & & Infinity & Infinity & & Infinity & Infinity & 0.000000 & & & & \\
\hline 1 & STOP & Standard $\mathbf{v}$ & pupil & Infinity & 0.000000 & & 145.500000 & 145.500000 & 0.000000 & & & & \\
\hline 2 & & Coordinate Break . & & & 0.000000 & - & 0.000000 & - & & 0.000000 & 0.000000 & 16.280000 & 0.000000 \\
\hline 3 & & Diffraction Grating & grating & Infinity & 0.000000 & & 167.385276 & - & 0.000000 & 1.475000 & -1.000000 & & \\
\hline 4 & & Coordinate Break & pupil relief & & $84.970000 \mathrm{~V}$ & & 0.000000 & - & & 0.000000 & 0.000000 & 16.270252 & 0.000000 \\
\hline 5 & & Even Asphere - & Lens 1 (Asph) & $-7.180000 E+04$ & 24.259997 & F_SILICA & 180.176027 & 188.422888 & 0.000000 & 0.000000 & $-1.413195 \mathrm{E}-09$ & $-6.067048 E-15$ & $-1.071633 \mathrm{E}-18$ \\
\hline 6 & & Standard $\mathbf{V}$ & & 382.860000 & 1.020000 & & 188.422888 & 188.422888 & 0.000000 & & & & \\
\hline 7 & & Standard $\mathbf{V}$ & Lens 2 & 374.890000 & 155.380000 & CAF2 & 189.970163 & 196.112719 & 0.000000 & & & & \\
\hline 8 & & Standard $\mathbf{v}$ & & -271.950000 & 0.640000 & & 196.112719 & 196.112719 & 0.000000 & & & & \\
\hline 10 & & Standard $\boldsymbol{V}$ & & 875.860000 & 1.000000 & & 212.503384 & 212.503384 & 0.000000 & & & & \\
\hline 11 & & Standard $\mathbf{V}$ & Lens 4 & 375.910000 & 179.300000 & CAF2 & 227.764641 & 227.764641 & 0.000000 & & & & \\
\hline 12 & & Standard $\mathbf{V}$ & & -354.740000 & 1.000000 & & 227.148153 & 227.764641 & 0.000000 & & & & \\
\hline 13 & & Even Asphere - & Lens 5 (Asph) & -577.130000 & 46.870000 & F_SILICA & 218.451797 & 218.451797 & 0.000000 & 0.000000 & $-1.043498 \mathrm{E}-09$ & $-2.161001 E-13$ & $4.018886 E-19$ \\
\hline 14 & & Standard $\mathbf{v}$ & & 367.100000 & 2.980000 & & $218.451797 \mathrm{P}$ & 218.451797 & 0.000000 & & & & \\
\hline 15 & & Standard & Lens 6 & 375.170000 & 178.130000 & CAF2 & $218.451797 P$ & 218.451797 & 0.000000 & & & & \\
\hline 16 & & Standard $\mathbf{v}$ & & -359.990000 & 59.770000 & & $218.451797 P$ & 218.451797 & 0.000000 & & & & \\
\hline 17 & & Even Asphere - & Lens 7 (Asph) & 247.120000 & 178.340000 & F_SILICA & 169.495810 & 169.495810 & 0.000000 & 0.000000 & $-2.987878 E-09$ & $8.528943 E-14$ & $1.436188 \mathrm{E}-18$ \\
\hline 20 & & Standard $\mathbf{V}$ & & 152.510000 & 0.000000 & & 74.297175 & 93.490148 & 0.000000 & & & & \\
\hline 21 & & Standard $\boldsymbol{v}$ & & Infinity & $41.520000 \mathrm{~V}$ & & 76.878286 & 76.878286 & 0.000000 & & & & \\
\hline 22 & & Coordinate Break V & & & 0.000000 & - & 0.000000 & . & & 0.000000 & 0.000000 & $0.000000 \mathrm{~V}$ & 0.000000 \\
\hline 23 & IMAGE & Standard $\mathbf{v}$ & & Infinity & . & & 64.234636 & 64.234636 & 0.000000 & & & & \\
\hline
\end{tabular}

Figure 10: Optical prescription for the 3Sph-5Asph design including the grating.
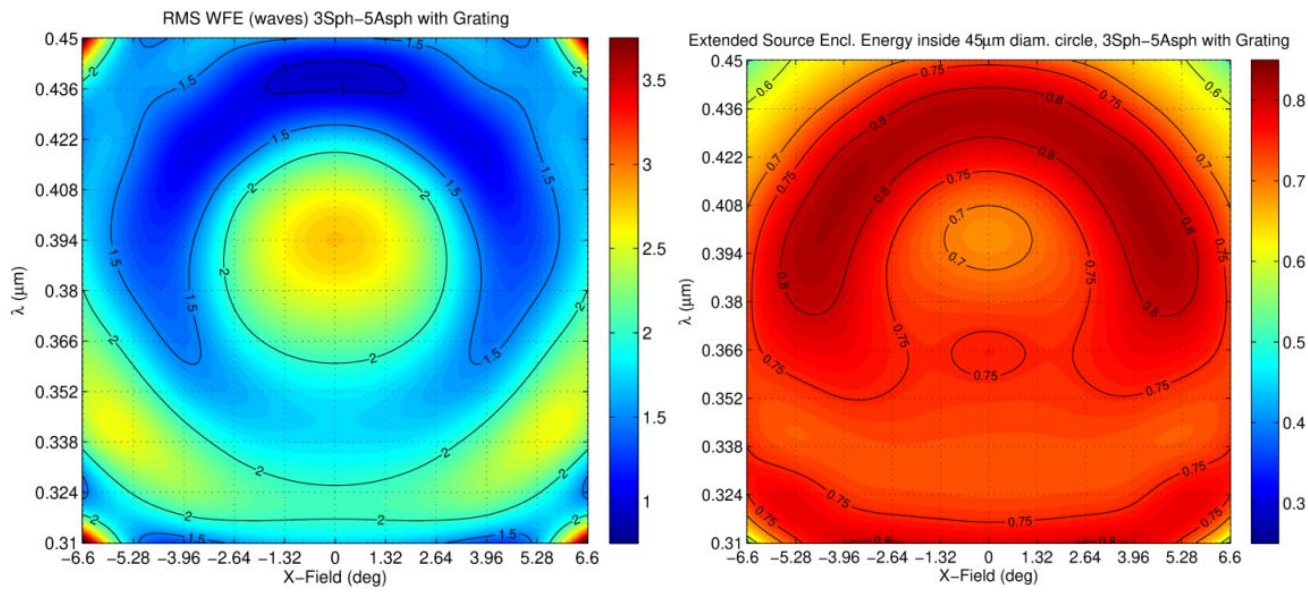

Figure 11: RMS WFE and extended source EE within $45 \mu \mathrm{m}$ diameter versus field and wavelength for the 8-element aspheric lens with grating. The RMS WFE map is plotted with color bar on a scale ranging from 0.75 to 3.75 waves, and the EE map from 0.25 to 0.85 .

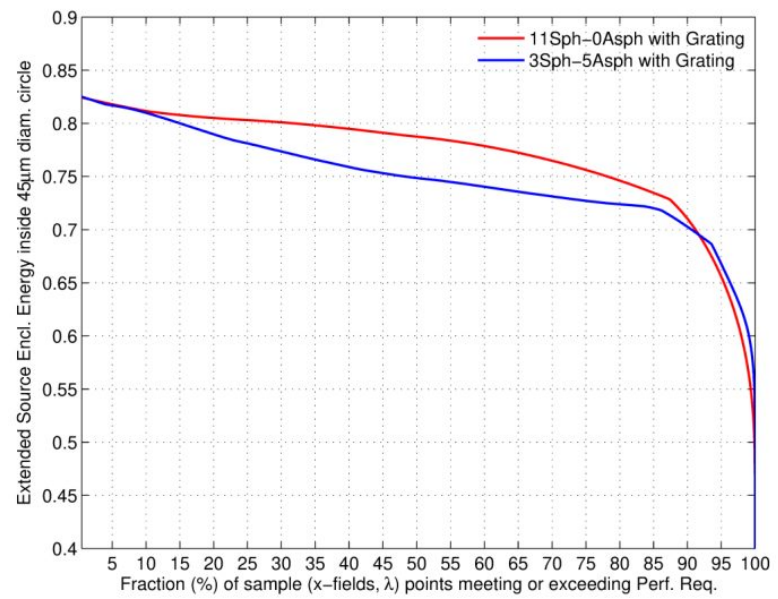

Figure 12: EE inside a 45micron diameter circle at the detector versus fraction of field and wavelength points meeting or exceeding a given performance level for the 11-element spherical lens with grating and the 8-element aspheric lens with grating. 


\subsubsection{Tolerance analysis}

Monte Carlo tolerance simulation results are displayed in Figure 13 for the same tolerances (see Table 1) and compensators (PR, BFD and image plane tilt) as for the spherical lens, 1000 simulation trials with uniform error statistics.

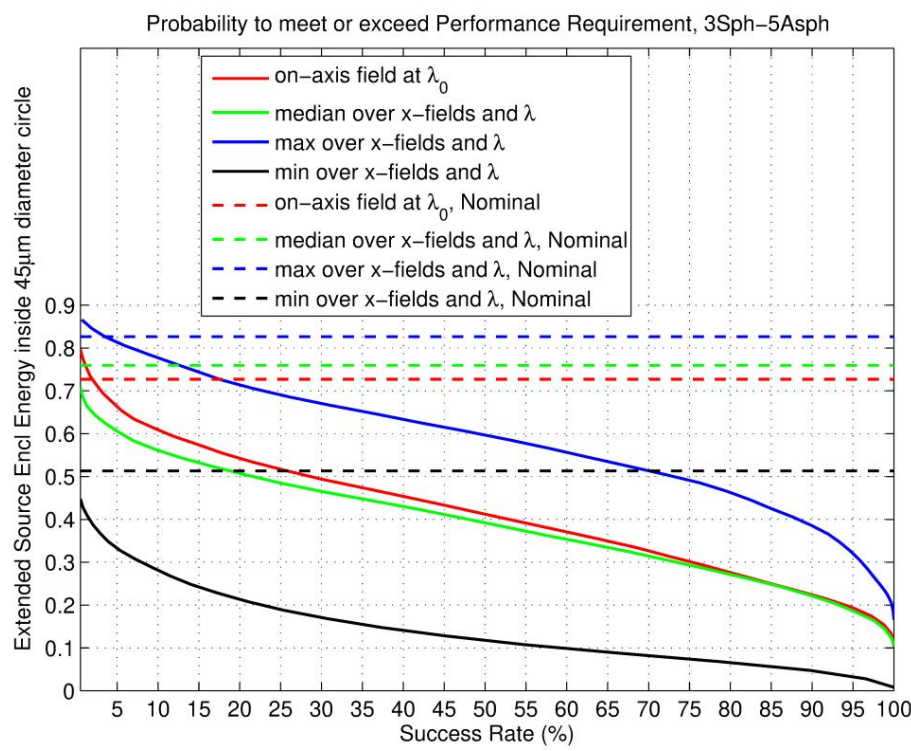

\begin{tabular}{|c|c|c|}
\hline \multicolumn{3}{|c|}{$\begin{array}{l}\text { Compensator statistics: } \\
\text { Thickness Surf } 4:\end{array}$} \\
\hline Nominal & : & 84.994799 \\
\hline Minimum & : & 79.830379 \\
\hline Maximum & $:$ & 100.041967 \\
\hline Mean & : & 84.463832 \\
\hline Standard Deviation & : & 1.344688 \\
\hline \multicolumn{3}{|l|}{ Thickness Surf 21: } \\
\hline Nominal & : & 41.519171 \\
\hline Minimum & : & 39.268311 \\
\hline Maximum & : & 44.128049 \\
\hline Mean & : & 41.511309 \\
\hline Standard Deviation & $:$ & 0.745127 \\
\hline \multicolumn{3}{|l|}{ Parameter 3 Surf 22: } \\
\hline Nominal & $:$ & -0.002236 \\
\hline Minimum & : & -0.515978 \\
\hline Maximum & : & 0.478932 \\
\hline Mean & : & -0.006207 \\
\hline Standard Deviation & : & 0.157612 \\
\hline \multicolumn{3}{|c|}{ Parameter 4 Surf 22: } \\
\hline Nominal & : & 0.000000 \\
\hline Minimum & : & -0.148676 \\
\hline Maximum & : & 0.156365 \\
\hline Mean & : & 0.000352 \\
\hline Standard Deviation & : & 0.050564 \\
\hline
\end{tabular}

Figure 13: Monte Carlo tolerance results of the aspheric lens with grating. 1,000 Monte Carlo trials were performed with uniform opto-mechanical error statistics. Success rate is defined as the probability over simulation trials to meet or exceed a given performance level. Right: Compensator statistics for the 3Sph-5Asph design including the grating. The first group is pupil relief, the second is BFD and last two are image plane tilt about the $\mathrm{x}$ - and $\mathrm{y}$-axis respectively.

\section{REFLECTIVE CAMERA DESIGN}

\subsection{Design progression}

Four- and five-mirror anastigmats (4MA, 5MA) have been discussed in the literature, for instance by Andrew Rackich [14] and Lacy Cook [15]. These imagers were invented to address fast and large field of view problems. Dave Shafer's high-performance 5MA design with external pupil served as our starting point [16]. It features a $300 \mathrm{~mm}$ EPD, a $22 \mathrm{deg}$ FoV diameter, a focal ratio $f / 2.0$ (600 mm focal length averaged over fields), pupil relief (EP-M2 distance) is $600 \mathrm{~mm}$ and image surface is flat. The surface sag of each mirror is described by an asymmetric general asphere [17]:

$$
\begin{aligned}
& z(x, y) \equiv \operatorname{sag}(x, y)=\sum_{i=0}^{65} z_{i}(x, y), \\
& z_{i}(x, y)=A_{i} \frac{x^{j(i)} y^{k(i)}}{R_{n}^{o}} \\
& o(i)=\text { floor }\left(\frac{\sqrt{1+8 i}-1}{2}\right), \quad k(i)=i-\frac{o(o+1)}{2}, \quad j(i)=o-k, \quad i(k, o)=k+\frac{o(o+1)}{2}
\end{aligned}
$$

where $i$ denotes the aspheric term number, $z_{i}$ denotes the sag of aspheric term number $i, o$ denotes the order of aspheric term $i, j$ is the algebraic power of the local $x$ coordinate on the surface, $k$ is the algebraic power of the local $y$ coordinate on the surface, and $R_{n}$ is the normalization radius. $x, y, z, A_{i}$ and $R_{n}$ all have millimetre units. The mirror system is symmetric about the $\mathrm{YZ}$ plane, therefore the aspheric coefficients $A_{i}$ are non-zero only for even powers of $x$ (odd powers of $x$ must be zero), which leads to a total 34 non-zero aspheric coefficients per mirror (order 10): order $o \geq 2$ has $N(o)=1+$ floor $(o / 2)$ non-zero terms, e.g. order 2 has 2 non-zero terms $\left(A_{3} x^{2} y^{0} / R_{n}^{2}\right),\left(A_{5} x^{0} y^{2} / R_{n}^{2}\right)$, order 3 
has 2 non-zero terms $\left(A_{7} x^{2} y^{1} / R_{n}^{3}\right),\left(A_{9} x^{0} y^{3} / R_{n}^{3}\right)$ etc. Even orders have only even powers of $x$ and even powers of $y$, whereas odd orders have only even powers of $x$ and odd powers of $y$. As a result, the OPD in the XP has zero projection onto Zernike modes with odd algebraic power in the cosine of the polar angle, i.e. Zernike modes proportional to $\cos (\phi), \cos ^{3}(\phi), \cos ^{5}(\phi), \cos ^{7}(\phi), \cdots$, i.e. modes proportional to odd algebraic powers of the $x$ coordinates in the XP. Although manufacturing and optical testing of large freeform mirrors remain challenging, promising progress in those areas has recently been made [18],[19],[20],[21],[22].

- Step1. The 5MA was scaled to the desired $392 \mathrm{~mm}$ focal length and $291 \mathrm{~mm}$ EPD. Focal length was determined using the Power Field method (ring of real rays traced around the chief ray of each field point). The 5MA was first analyzed without the grating at the pupil plane and with FoV discretized into a $7 \times 7$ array of field points with $\theta_{y}=\left\{0^{\circ}, \pm 2.1^{\circ}, \pm 4.2^{\circ}, \pm 6.3^{\circ}\right\}$ and $\theta_{x}=\left\{0^{\circ}, 1^{\circ}, 2^{\circ}, 3^{\circ}, 4^{\circ}, 5^{\circ}, 6.6^{\circ}\right\}$ to cover half of $\mathrm{FoV}_{x}$ given in (1.16) and $\mathrm{FoV}_{y}$ given in (1.5). After optimization (62x62 rectangular array pupil discretization, RMS WFE optimization criterion, local DLS), the desired first-order properties were met but interferences between M1/M2, M3/M5, M2/M4, M1/M3, and M4/IMA were observed. To overcome the clearance issue, a set of weighted constraints was imposed on ray global Y-heights in the Merit Function:

- Lowest point on M3 above highest point on M5,

- Lowest point on M1 above highest point on M2,

- Lowest point on M2 above highest point on M4,

- Lowest point on M1 above highest point on M3,

- Lowest point on M4 above highest point on IMA.

- Step2. After optimization of mirror spacings, X-tilts and aspheric coefficients, the number of non-zero aspheric coefficients per mirror was reduced to only 23 (orders 2 to 8 ) without performance loss.

- Step3. The grating was introduced at the pupil, $11 \mathrm{X}$-fields and 11 wavelengths were simulated, the MF was updated and the system was re-optimized.

\subsection{Performance results}

\subsubsection{Nominal performance}

In this Section, performance of the 5-mirror anastigmat (5MA) with the grating and fibers in the pseudo-slit is discussed. The optical layout is show in Figure 14, the optical prescription in Figure 15 and performance results in Figure 16 and Figure 17. The merit function was built with uniform $x$-fields and wavelengths weights and optimization was performed using local DLS. All sample points have between $92 \%$ and $98 \%$ extended source enclosed energy within a 45microns diameter circle. Focus sensitivity and a field distortion map are displayed in Figure 18. The map was obtained by removing the diffraction grating. Maximum distortion is only $-1.85 \%$. Note that field distortion is not symmetric with respect to Y. Note also that the excellent imaging performance comes at the expense of poor intermediate pupil (located between M4 and M5) quality, but this is not of concern in spectrograph applications. No attempt has been done to optimize both image and pupil quality. In other applications (e.g. microlithography), variation in relative illumination across the image may have to be kept very low, therefore pupil size variations across the field may not be allowed and tight pupil size and pupil shape control may be required. 


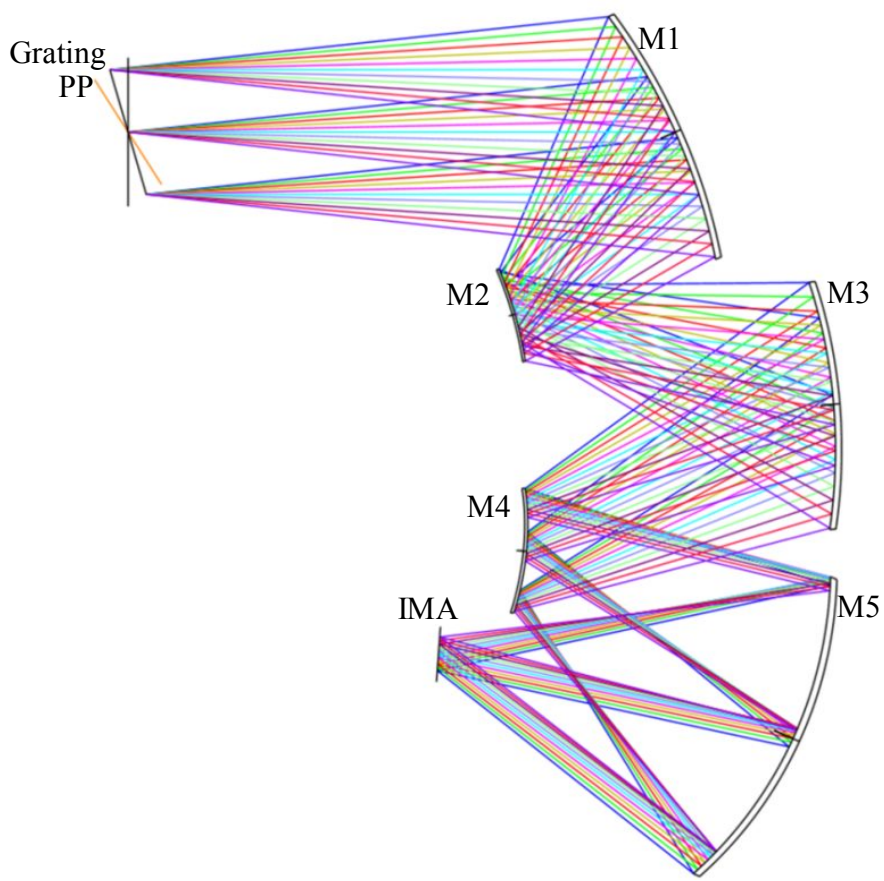

Figure 14: Optical layout and on-axis field Y-fan for the 5MA design including the grating. Short/long wavelength rays leave the pupil with positive/negative slope. The global coordinate reference surface is the vertex plane of the first mirror. Rays are colored by wavelength.

\begin{tabular}{|c|c|c|c|c|c|c|c|c|c|c|c|c|}
\hline \multirow{2}{*}{$0_{0}^{4}$} & \multicolumn{2}{|r|}{ Surt:Type } & \multirow[t]{2}{*}{ Comment } & \multirow{2}{*}{$\begin{array}{l}\text { Radius } \\
\text { Infinity }\end{array}$} & \multirow{2}{*}{$\begin{array}{r}\text { Thickness } \\
\text { Infinity }\end{array}$} & \multirow[t]{2}{*}{ Material } & \multirow{2}{*}{$\begin{array}{c}\text { Semi-Diameter } \\
\text { Infinity }\end{array}$} & \multirow[t]{2}{*}{ Decenter X } & \multirow[t]{2}{*}{ Decenter $Y$} & Tilt About X & Tilt About Y & \multirow[t]{2}{*}{ Tilt About Z } \\
\hline & OBJECT & Standard $\mathbf{v}$ & & & & & & & & & & \\
\hline 1 & STOP & Standard $\mathbf{v}$ & & Infinity & 0.000000 & & $145.500000 \mathrm{U}$ & & & & & \\
\hline 2 & & Coordinate Break - & & & 0.000000 & 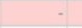 & 0.000000 & 0.000000 & 0.000000 & 32.560000 & 0.000000 & 0.000000 \\
\hline 3 & & Standard $\mathbf{v}$ & & Infinity & 0.000000 & & 173.733767 & & & & & \\
\hline 4 & & Coordinate Break - & & & 0.000000 & - & 0.000000 & 0.000000 & 0.000000 & -32.560000 & 0.000000 & 0.000000 \\
\hline 5 & & Coordinate Break - & & & 0.000000 & - & 0.000000 & 0.000000 & 0.000000 & 16.280000 & 0.000000 & 0.000000 \\
\hline 6 & & Diffraction Grating & grating & Infinity & 0.000000 & & 152.466859 & 1.475000 & -1.000000 & & & \\
\hline 7 & & Coordinate Break - & pupil relief & & $1622.506756 \mathrm{~V}$ & - & 0.000000 & 0.000000 & 0.000000 & 16.270252 & 0.000000 & 0.000000 \\
\hline 8 & & Standard $\mathbf{v}$ & & Infinity & 0.000000 & & 405.068419 & & & & & \\
\hline 9 & & Coordinate Break - & & & 0.000000 & & 0.000000 & 0.000000 & 0.000000 & -21.937250 & 0.000000 & 0.000000 \\
\hline 10 & & Extended Polynomial - & M1 & Infinity & 0.000000 & MIRROR & 412.151262 & & & & & \\
\hline 11 & & Coordinate Break - & & & $-670.173718 \mathrm{~V}$ & - & 0.000000 & 0.000000 & 0.000000 & -21.937250 & 0.000000 & 0.000000 \\
\hline 12 & & Coordinate Break - & & & 0.000000 & - & 0.000000 & 0.000000 & 0.000000 & 29.872204 & 0.000000 & 0.000000 \\
\hline 13 & & Extended Polynomial - & M2 & Infinity & 0.000000 & MIRROR & 165.181792 & & & & & \\
\hline 14 & & Coordinate Break - & & & $861.669272 \mathrm{~V}$ & - & 0.000000 & 0.000000 & 0.000000 & 29.872204 & 0.000000 & 0.000000 \\
\hline 15 & & Coordinate Break - & & & 0.000000 & - & 0.000000 & 0.000000 & 0.000000 & -21.549102 & 0.000000 & 0.000000 \\
\hline 16 & & Extended Polynomial - & M3 & Infinity & 0.000000 & MIRROR & 368.263766 & & & & & \\
\hline 17 & & Coordinate Break - & & & $-890.297769 \mathrm{~V}$ & - & 0.000000 & 0.000000 & 0.000000 & -21.549102 & 0.000000 & 0.000000 \\
\hline 18 & & Coordinate Break - & & & 0.000000 & - & 0.000000 & 0.000000 & 0.000000 & 26.648513 & 0.000000 & 0.000000 \\
\hline 19 & & Extended Polynomial - & M4 & Infinity & 0.000000 & MIRROR & 173.437391 & & & & & \\
\hline 20 & & Coordinate Break - & & & $836.061098 \mathrm{~V}$ & - & 0.000000 & 0.000000 & 0.000000 & 26.648513 & 0.000000 & 0.000000 \\
\hline 21 & & Coordinate Break - & & & 0.000000 & - & 0.000000 & 0.000000 & 0.000000 & -9.757689 & 0.000000 & 0.000000 \\
\hline 22 & & Extended Polynomial - & M5 & Infinity & 0.000000 & MIRROR & 414.804641 & & & & & \\
\hline 23 & & Coordinate Break - & & & $-777.913708 \mathrm{~V}$ & - & 0.000000 & 0.000000 & 0.000000 & -9.757689 & 0.000000 & 0.000000 \\
\hline 24 & & Standard $\mathbf{v}$ & & Infinity & $-94.534669 \mathrm{~V}$ & & $0.000000 \mathrm{U}$ & & & & & \\
\hline 25 & & Coordinate Break - & & & 0.000000 & - & 0.000000 & 0.000000 & 0.000000 & -4.892571 & 0.000000 & 0.000000 \\
\hline 26 & IMAGE & Standard $\mathbf{v}$ & & Infinity & - & & 62.626054 & & & & & \\
\hline
\end{tabular}

Figure 15: Optical prescription for the 5MA design including the grating. 

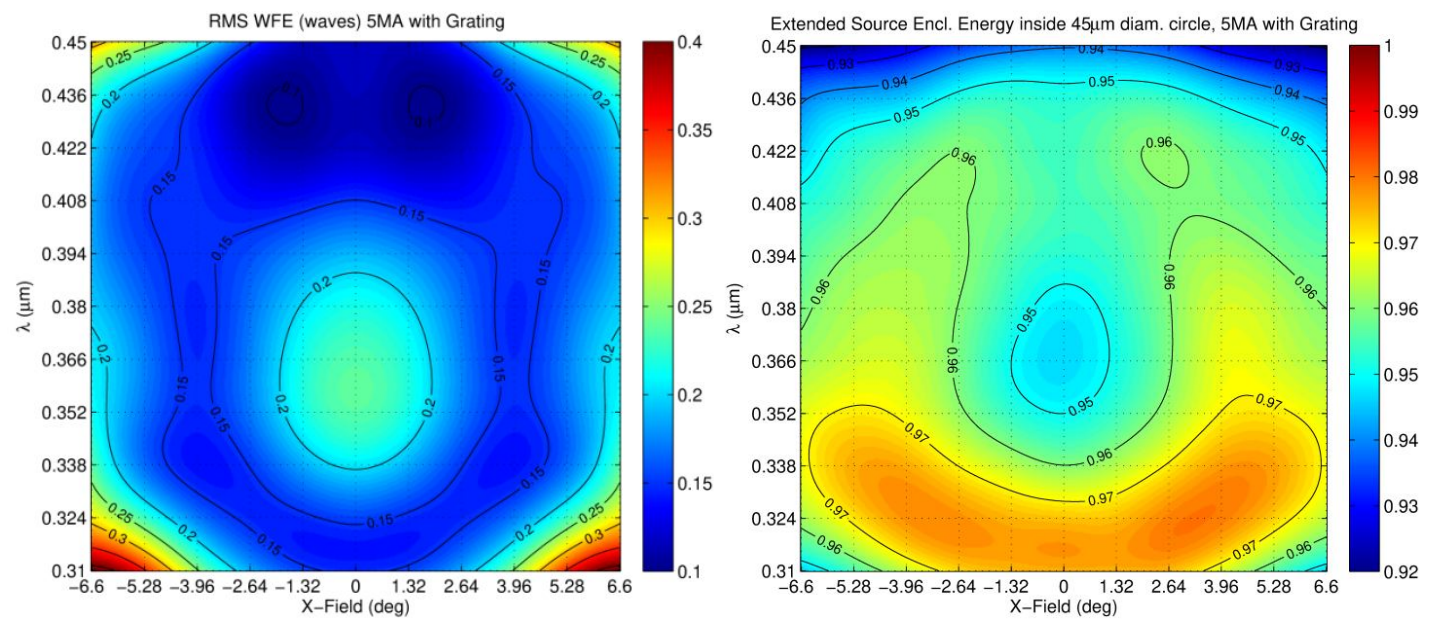

Figure 16: RMS WFE and extended source EE within $45 \mu \mathrm{m}$ diameter versus field and wavelength for the 5MA with grating.

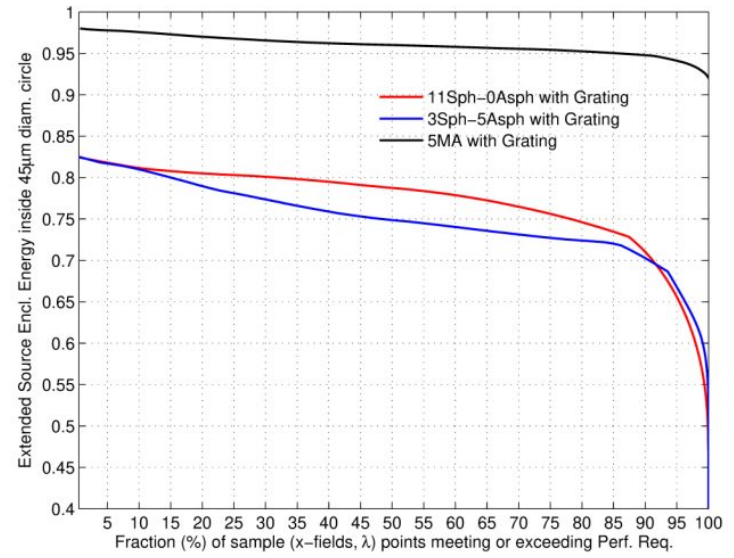

Figure 17: EE inside a 45micron diameter circle at the detector versus fraction of field and wavelength points meeting or exceeding a given performance level for the 11-element spherical lens with grating, the 8-element aspheric lens with grating, and the 5MA with grating.
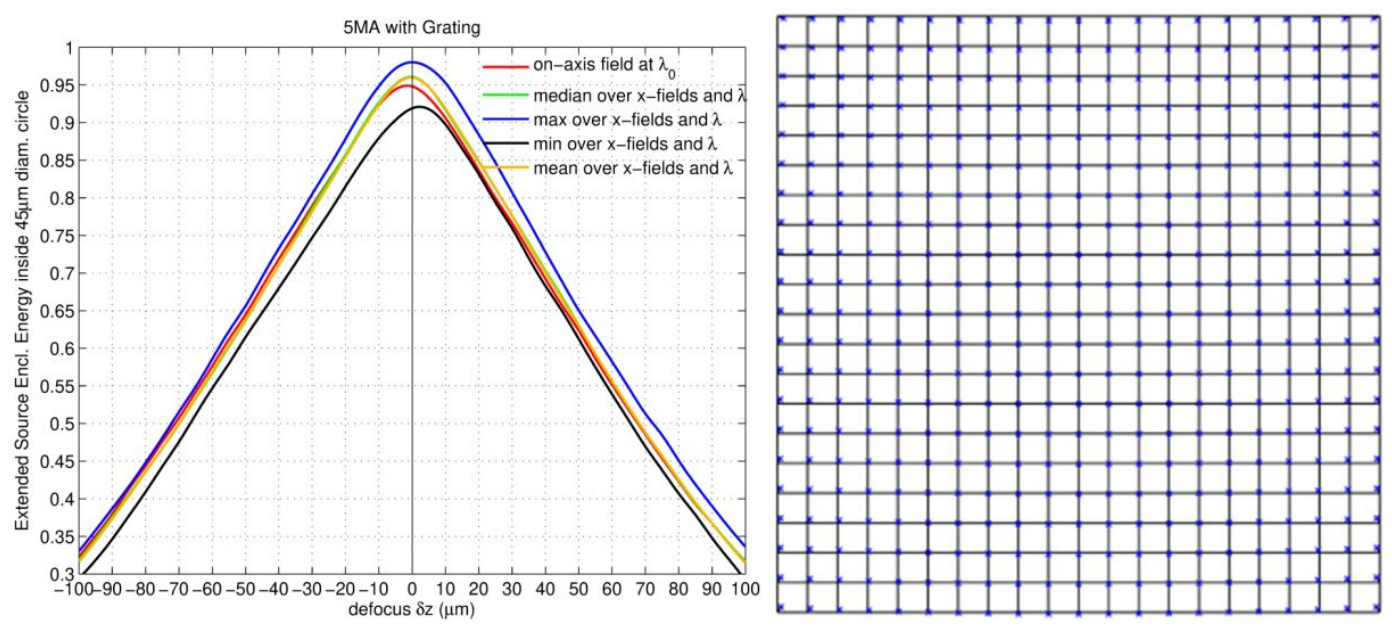

Figure 18: Left: Extended source EE within $45 \mu \mathrm{m}$ diameter versus defocus from nominal image plane position for the $5 \mathrm{MA}$ with grating. Right: Field distortion map for the 13.2deg (spatial direction) x 12.6deg (dispersion direction) FoV (1x scale factor), 92.6mm $\mathrm{x} 83.5 \mathrm{~mm}$. Maximum distortion is only $-1.85 \%$. 


\subsubsection{Tolerance analysis}

The tolerances listed in Table 2 were imposed on the freeform 5MA (pending mechanical review).

\begin{tabular}{|l|l|l|}
\hline Item & Value & Comment \\
\hline Thickness (air) & $\pm 100 \mu \mathrm{m}$ & \\
\hline $\mathrm{X}$ and Y element decenter & $\pm 50 \mu \mathrm{m}$ & $\begin{array}{l}\text { twice Optimax high-precision thickness } \\
\text { tolerance as an estimate for linear distance } \\
\text { tolerance, pending mechanical review }\end{array}$ \\
\hline Surface irregularity & $\begin{array}{l} \pm 0.02 \% \text { of the nominal } \\
\text { coefficient value for } \\
\mathrm{M} 1 / \mathrm{M} 3 / \mathrm{M} 4, \pm 0.1 \% \text { of the } \\
\text { nominal value for M2 and } \\
\pm 0.003 \% \text { of the nominal value } \\
\text { for M5. }\end{array}$ & $\begin{array}{l}\text { Parabolic terms only: } \\
\left(A_{3} x^{2} y^{0} / R_{n}^{2}\right),\left(A_{5} x^{0} y^{2} / R_{n}^{2}\right) \text { Tolerances were } \\
\text { obtained from inverse sensitivity analysis } \\
\text { (nominal MF value was 2.08 and was allowed } \\
\text { to increase up to 2.68). As shown in Table 3 } \\
\text { these tolerances also correspond to the change } \\
\text { in radius of curvature. }\end{array}$ \\
\hline Element X and Y tilt & $\pm 0.0083 \mathrm{deg}( \pm 0.5$ arcmin $)$ & Optimax wedge prism high precision \\
\hline
\end{tabular}

Table 2: Opto-mechanical tolerances adopted for the freeform 5MA tolerance analysis.

Four active compensation strategies were studied in 600 Monte Carlo simulation trials with uniform opto-mechanical error statistics:

- Active BFD adjustment (constrained between 50mm and 200mm),

- Active Pupil Relief adjustment (constrained between $1422 \mathrm{~mm}$ and $1822 \mathrm{~mm}$ ),

- $\quad$ Active detector plane X- and Y-tilt adjustment (constrained between -15deg and +10deg).

Radii of curvature $R_{x}$ and $R_{y}$ along the x- and y-directions depend solely on the quadratic sag coefficients $A_{3}$ and $A_{5}$ respectively. The x-cross section of the parabolic sag component is given by $z_{3}=A_{3} \frac{x^{2}}{R_{n}^{2}}=\frac{c_{x} x^{2}}{2}$ where the last equality is obtained from the sag expression of a parabola $(K=-1)$ and $c_{x}=1 / R_{x}$ is x-curvature and $R_{x}$ the $\mathrm{x}$-radius of curvature. We thus have $R_{x}=\frac{R_{n}^{2}}{2 A_{3}}=-2 f_{x}$ where $f_{x}$ denotes the focal length for the x-direction. Denoting by $z_{3}{ }^{\prime}=A_{3}{ }^{\prime} \frac{x^{2}}{R_{n}^{2}}=\frac{c_{x}{ }^{\prime} x^{2}}{2}$ the parabolic sag along the x-direction for the perturbed coefficient $A_{3}{ }^{\prime}=A_{3}+\operatorname{tol}_{3}$, we have $R_{x}{ }^{\prime}=\frac{R_{n}^{2}}{2 A_{3}{ }^{\prime}}$ and:

$$
\begin{aligned}
& \frac{c_{x}}{c_{x}{ }^{\prime}}=\frac{R_{x}{ }^{\prime}}{R_{x}}=\frac{f_{x}{ }^{\prime}}{f_{x}}=\frac{A_{3}}{A_{3}{ }^{\prime}}=\frac{1}{1+\frac{\mathrm{tol}_{3}}{A_{3}}} \\
& z_{3}{ }^{\prime}\left(x_{\max }\right)-z_{3}\left(x_{\max }\right)=\left(A_{3}{ }^{\prime}-A_{3}\right) \frac{x_{\max }^{2}}{R_{n}^{2}}=\operatorname{tol}_{3} \frac{x_{\max }^{2}}{R_{n}^{2}}
\end{aligned}
$$

Similar equations apply to the parabolic term along the y-direction $z_{5}(y)=A_{5} \frac{y^{2}}{R_{n}^{2}}=\frac{c_{y} y^{2}}{2}$. Table 3 presents the calculations performed to estimate the change in radius of curvature and the change in parabolic sag when the above tolerances are applied. It is worth noting that the change in radius of curvature (or equivalently focal length) is on the order of only $0.02 \%$ at the exception of M2 which has a relaxed radius tolerance of $0.1 \%$ and M5 with a tightened radius 
tolerance of $0.03 \%$. Figure 19 shows performance versus success rate (confidence level) for 600 Monte Carlo trials when the above opto-mechanical errors are applied with uniform statistics.

\begin{tabular}{|c|c|c|c|c|c|c|}
\hline & M1 & M2 & M3 & M4 & M5 & \multirow{2}{*}{\begin{tabular}{|l}
\multicolumn{2}{c}{ from lens data and tol data } \\
R_n
\end{tabular}} \\
\hline normalization radius $(\mathrm{mm})$ & \multicolumn{5}{|c|}{0.643279} & \\
\hline Ray X Max (mm) & 336.7591 & 114.9712 & 299.9902 & 146.5281 & 350.5993 & $\mathrm{x}$ (from footprint) \\
\hline $\mathrm{A} 3(\mathrm{~mm})$ & $\mid-1.480454 \mathrm{E}-04$ & $-2.720384 \mathrm{E}-04$ & $-1.561555 E-04$ & $\mid-2.729770 E-04$ & $-2.024802 E-04$ & $\left(x / R \_n\right)^{\wedge} 2$ coefficient \\
\hline Nominal X Radius of Curvature $(\mathrm{mm})$ & -1397.571 & -760.569 & -1324.987 & -757.954 & -1021.848 & $R=\left(R_{\_} n\right)^{\wedge} 2 /\left(2^{*} A\right)$ \\
\hline Nominal X Parabolic Sag (mm) & -40.5728 & -8.6898 & -33.9604 & -14.1635 & -60.1459 & $z=A^{*}\left(x / R \_n\right)^{\wedge} 2$ \\
\hline $\min$ tol3 $(\mathrm{mm})$ & \begin{tabular}{|l|}
$-3.538548 E-08$ \\
\end{tabular} & $-2.700000 \mathrm{E}-07$ & $-4.956410 \mathrm{E}-08$ & $\mid-6.196211 E-08$ & $-6.910522 \mathrm{E}-09$ & tol \\
\hline $\min$ tol3 / A3 & $2.390178 \mathrm{E}-04$ & $9.925069 \mathrm{E}-04$ & $3.174022 \mathrm{E}-04$ & $2.269866 \mathrm{E}-04$ & 3.412937E-05 & tol/A \\
\hline $\mathrm{A} 3^{\prime}(\mathrm{mm})$ & $\mid-1.480808 E-04$ & $-2.723084 \mathrm{E}-04$ & $-1.562051 \mathrm{E}-04$ & $\mid-2.730390 \mathrm{E}-04$ & $-2.024871 \mathrm{E}-04$ & $A^{\prime}=A+$ tol \\
\hline Perturbed X Radius of Curvature $(\mathrm{mm})$ & -1397.237 & -759.815 & -1324.566 & -757.782 & -1021.813 & $R^{\prime}=\left(R_{-} n\right)^{\wedge} 2 /\left(2^{*} A^{\prime}\right)$ \\
\hline Perturbed X Parabolic Sag (mm) & -40.5825 & -8.6984 & -33.9712 & -14.1667 & -60.1479 & $z^{\prime}=A^{\prime *}\left(x / R_{\_} n\right)^{\wedge} 2$ \\
\hline Change in X Radius of Curvature (\%) & 0.02 & 0.10 & 0.03 & 0.02 & 0.003 & $\left(\mathrm{R}-\mathrm{R}^{\prime}\right) / \mathrm{R}^{*} 100=(\mathrm{tol} / \mathrm{A}) /[1+(\mathrm{tol} / \mathrm{A})]^{*} 100$ \\
\hline Change in X Parabolic Sag (um) & 9.698 & 8.625 & 10.779 & 3.215 & 2.053 & $\left(z-z^{\prime}\right)^{*} 1000=-$ tol $^{*}\left(x / R \_n\right)^{\wedge} 2^{*} 1000$ \\
\hline Change in X Parabolic OPD (um) & 19.395 & 17.249 & 21.558 & 6.430 & 4.105 & $2^{*}\left(z-z^{\prime}\right) * 1000$ \\
\hline Change in X Parabolic OPD (waves @ 0.5um) (Nb. of fringes) & 39 & 34 & 43 & 13 & 8 & $2 *\left(z-z^{\prime}\right) * 1000 / w v l$ \\
\hline $\operatorname{Ray} Y \operatorname{Max}(\mathrm{mm})$ & 344.1008 & 111.2554 & 274.4203 & 138.3533 & 357.0724 & y (from footprint) \\
\hline A5 $(\mathrm{mm})$ & $\mid-1.264072 \mathrm{E}-04$ & $-1.871564 \mathrm{E}-04$ & $-1.344174 E-04$ & $\mid-2.337881 E-04$ & $-1.988710 \mathrm{E}-04$ & $\left(y / R_{-} n\right)^{\wedge} 2$ coefficient \\
\hline Nominal Y Radius of Curvature $(\mathrm{mm})$ & -1636.805 & -1105.514 & -1539.265 & -885.006 & -1040.393 & $R=\left(R_{-} n\right)^{\wedge} 2 /\left(2^{*} A\right)$ \\
\hline Nominal Y Parabolic Sag (mm) & -36.1697 & -5.5982 & -24.4618 & -10.8144 & -61.2753 & $z=A^{*}\left(x / R_{-} n\right)^{\wedge} 2$ \\
\hline $\min$ tol5 $(\mathrm{mm})$ & $\mid-3.403932 E-08$ & $-1.800000 \mathrm{E}-07$ & $-4.629989 \mathrm{E}-08$ & $-5.536229 \mathrm{E}-08$ & $-6.716893 \mathrm{E}-09$ & tol \\
\hline $\min$ tol5 / A5 & $2.692831 \mathrm{E}-04$ & $9.617625 \mathrm{E}-04$ & $3.444486 \mathrm{E}-04$ & $2.368054 \mathrm{E}-04$ & $3.377513 \mathrm{E}-05$ & tol/A \\
\hline A5' (mm) & $\mid-1.264412 \mathrm{E}-04$ & $-1.873364 \mathrm{E}-04$ & $-1.344637 E-04$ & $\mid-2.338435 E-04$ & $-1.988777 \mathrm{E}-04$ & $A^{\prime}=A+$ tol \\
\hline Perturbed Y Radius of Curvature (mm) & -1636.364 & -1104.451 & -1538.735 & -884.797 & -1040.358 & $R^{\prime}=\left(R \_n\right)^{\wedge} 2 /\left(2^{*} A^{\prime}\right)$ \\
\hline Perturbed Y Parabolic Sag (mm) & -36.1794 & -5.6036 & -24.4703 & -10.8170 & -61.2773 & $z^{\prime}=A^{\prime *}\left(y / R_{-} n\right)^{\wedge} 2$ \\
\hline Change in Y Radius of Curvature (\%) & 0.03 & 0.10 & 0.03 & 0.02 & 0.003 & $\left(R-R^{\prime}\right) / R^{*} 100=($ tol $/ A) /[1+(\text { tol/A })]^{*} 100$ \\
\hline Change in Y Parabolic Sag (um) & 9.740 & 5.384 & 8.426 & 2.561 & 2.070 & $\left(z-z^{\prime}\right)^{*} 1000=-t o l^{*}\left(y / R \_n\right)^{\wedge} 2^{*} 1000$ \\
\hline Change in Y Parabolic OPD (um) & 19.480 & 10.768 & 16.852 & 5.122 & 4.139 & $2 *\left(z-z^{\prime}\right) * 1000$ \\
\hline Change in Y Parabolic OPD (waves @ 0.5um) (Nb. of fringes) & 39 & 22 & 34 & 10 & 8 & $2 z^{*}\left(z-z^{\prime}\right) * 1000 / w v l$ \\
\hline
\end{tabular}

Table 3: Change in radius of curvature and defocus OPD for each mirror of the 5MA with grating when tolerances listed in Table 2 are applied to the nominal parabolic X- and Y-sag coefficients.

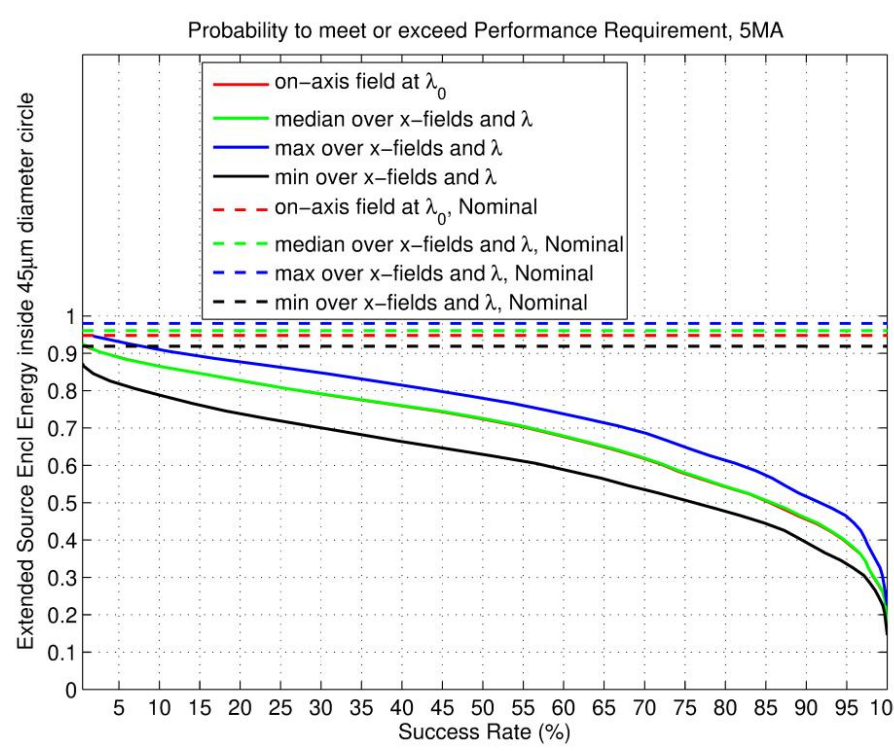

\begin{tabular}{|c|c|c|}
\hline \multicolumn{3}{|c|}{$\begin{array}{l}\text { Compensator Statistics: } \\
\text { Thickness Surf } 7:\end{array}$} \\
\hline Nominal & $:$ & 1620.625688 \\
\hline Minimum & : & 1421.618555 \\
\hline Maximum & $:$ & 1780.735855 \\
\hline Mean & : & 1617.167349 \\
\hline Standard Deviation & : & 81.790369 \\
\hline \multicolumn{3}{|l|}{ Thickness Surf 24: } \\
\hline Nominal & : & -94.534680 \\
\hline Minimum & : & -94.599436 \\
\hline Maximum & : & -94.474328 \\
\hline Mean & $:$ & -94.534866 \\
\hline Standard Deviation & : & 0.019247 \\
\hline \multicolumn{3}{|l|}{ Parameter 3 Surf 25: } \\
\hline Nominal & : & -4.892592 \\
\hline Minimum & : & -4.919168 \\
\hline Maximum & : & -4.873219 \\
\hline Mean & : & -4.892847 \\
\hline Standard Deviation & : & 0.008029 \\
\hline \multicolumn{3}{|c|}{ Parameter 4 Surf 25: } \\
\hline Nominal & : & -0.000000 \\
\hline Minimum & : & -0.015629 \\
\hline Maximum & : & 0.015629 \\
\hline Mean & $:$ & -0.000000 \\
\hline Standard Deviation & : & 0.004569 \\
\hline
\end{tabular}

Figure 19: Monte Carlo tolerance results of the 5MA with grating. 600 Monte Carlo trials were performed with uniform optomechanical error statistics. Success rate is defined as the probability over simulation trials to meet or exceed a given performance level. Right: compensator statistics for the 5MA design including the grating. The first group is pupil relief, the second is BFD and last two are image plane tilt about the $\mathrm{x}$ - and $\mathrm{y}$-axis respectively.

\section{CATADIOPTRIC CAMERA DESIGN}

\subsection{Design progression}

Given the complexity of the refractive and reflective designs discussed, we deemed important to seek simpler solutions [23]. In this regard, an analysis of Bernard Delabre's compact, fast, 3-element, catadioptric camera [24] has been performed. The camera was recently developed for the MOONS spectrograph of the VLT. First-order MOONS 
parameters are close to the latest fiber WFOS parameters. The camera design consists of an aspheric concave mirror and an aspheric fused silica meniscus corrector lens glued to an aspheric fused silica convex-plano field flattener lens. Only the front surface of the corrector and field flattener lens is aspheric. Winlight [25] can manufacture such cameras using Luphos GmbH metrology equipment [26], which allows manufacturing of highly aspheric lenses and mirrors. The camera is extremely fast with a focal ratio of $\mathrm{f} / 0.95$, it has an external pupil of diameter equal to $D_{\mathrm{EP}}=280 \mathrm{~mm}$ (focal length equal to $f_{\text {cam }}=266 \mathrm{~mm}$ ), and can accommodate a $4 \mathrm{~K}$ detector with $15 \mu \mathrm{m}$ pixels (image width equal to $\left.l_{y}=l_{x}=61 \mathrm{~mm}\right)$, i.e. $\mathrm{FoV}=\mathrm{FoV}_{y}=\mathrm{FoV}_{x}=2 \cdot \operatorname{atan}\left(\frac{l_{y}}{2 f_{\text {cam }}}\right) \cdot \frac{180}{\pi} \mathrm{deg}=13.1 \mathrm{deg}$. The main drawback of the camera is the vignetting due to the detector central obscuration. A good approximation for the vignetting amount is given by the ratio of the circular image area at the detector to the circular entrance pupil area. This ratio depends only on focal ratio and field of view and is given by:

$$
\mathrm{v}=\left(\frac{2 f_{\mathrm{cam}} \tan (\mathrm{FoV} / 2)}{D_{\mathrm{EP}}}\right)^{2}=4 f_{\#}^{2} \tan ^{2}(\mathrm{FoV} / 2)
$$

For the MOONS camera's focal ratio and field of view, (1.23) yields $20 \%$ vignetting.

- The design was scaled to the desired $392 \mathrm{~mm}$ focal length and $291 \mathrm{~mm}$ EPD. The grating was introduced at the pupil, the MF was setup with $11 \mathrm{X}$-fields and 11 wavelengths, and the system was re-optimized. For the focal ratio given in (1.15) and field of view given in (1.18), (1.23) yields also 20\% vignetting. Speeding up the camera to f $\mid 1.17$ while keeping the same FoV would reduce vignetting to $15 \%$.

\subsection{Performance results}

\subsubsection{Nominal performance}

In this Section, performance of the 3-element catadioptric camera with the grating and fibers in the pseudo-slit is discussed. The optical layout is show in Figure 20, the optical prescription in Figure 21 and performance results in Figure 22 and Figure 23. The mirror (M1) has a diameter of 480mm.

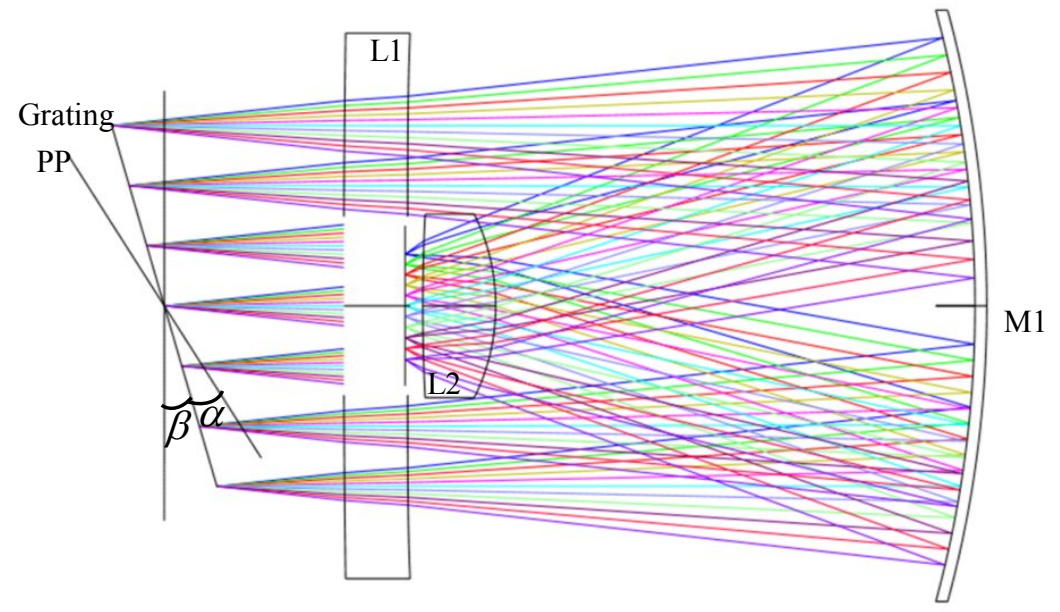

Figure 20: Optical layout and on-axis field Y-fan for the 3-element catadioptric design including the grating. Short/long wavelength rays leave the pupil with positive/negative slope. The global coordinate reference surface is the vertex plane of the first lens. Rays are colored by wavelength 


\begin{tabular}{|c|c|c|c|c|c|c|c|c|c|c|c|c|c|}
\hline 4 & & Surf:Type & Comment & Radius & Thickness & Material & Clear Semi-Dia & Mech Semi-Dia & Conic & 2nd Order Ter & 4th Order Term & 6th Order Term & 8th Order Term \\
\hline 0 & OBJECT & Standard $=$ & & Infinity & Infinity & & Infinity & Infinity & 0.000000 & & & & \\
\hline 1 & STOP & Standard $\mathbf{v}$ & & Infinity & 0.000000 & & 145.500000 & 145.500000 & 0.000000 & & & & \\
\hline 2 & & Coordinate Break - & & & 0.000000 & - & 0.000000 & - & & 0.000000 & 0.000000 & 32.560000 & 0.000000 \\
\hline 3 & & Standard $\mathbf{V}$ & & Infinity & 0.000000 & & 173.352523 & 173.352523 & 0.000000 & & & & \\
\hline 4 & & Standard $\mathbf{v}$ & & Infinity & 0.000000 & & 173.352523 & 173.352523 & 0.000000 & & & & \\
\hline 5 & & Coordinate Break - & & & 0.000000 & - & 0.000000 & - & & 0.000000 & 0.000000 & -32.560000 & 0.000000 \\
\hline 6 & & Coordinate Break - & & & 0.000000 & - & 0.000000 & - & & 0.000000 & 0.000000 & 16.280000 & 0.000000 \\
\hline 7 & & Diffraction Grating & grating & Infinity & 0.000000 & & 152.168433 & - & 0.000000 & 1.475000 & -1.000000 & & \\
\hline 8 & & Coordinate Break - & pupil relief & & $161.066952 \mathrm{~V}$ & - & 0.000000 & - & & 0.000000 & 0.000000 & 16.270252 & 0.000000 \\
\hline 10 & (aper) & Even Asphere ${ }^{-}$ & & $-3.789523 E+04$ & 457.993123 & & 179.505379 & 220.000000 & 0.000000 & 0.000000 & $9.582073 E-10$ & $2.298253 E-15$ & 0.000000 \\
\hline 11 & & Even Asphere - & & -919.432598 & -386.977906 & MIRROR & 240.726668 & 240.726668 & 0.000000 & 0.000000 & $-5.918060 \mathrm{E}-11$ & $-2.663039 \mathrm{E}-17$ & 0.000000 \\
\hline 12 & (aper) & Even Asphere ${ }^{-}$ & & -169.679882 & -58.855147 & CAF2 & $74.000000 \mathrm{U}$ & 74.000000 & 0.000000 & 0.000000 & $-1.132239 \mathrm{E}-08$ & $1.051974 E-13$ & 0.000000 \\
\hline 13 & (aper) & Standard $\mathbf{v}$ & & 1430.802603 & 0.000000 & & $74.000000 \mathrm{P}$ & 74.000000 & 0.000000 & & & & \\
\hline 14 & & Standard $\mathbf{V}$ & & Infinity & $-14.299155 \mathrm{~V}$ & & 74.800744 & 74.800744 & 0.000000 & & & & \\
\hline 15 & & Coordinate Break . & & & 0.000000 & - & 0.000000 & - & & 0.000000 & 0.000000 & $9.559910 \mathrm{E}-04 \mathrm{~V}$ & $4.018475 \mathrm{E}-03$ \\
\hline 16 & IMAGE & Standard $\mathbf{V}$ & & Infinity & - & & 64.633432 & 64.633432 & 0.000000 & & & & \\
\hline
\end{tabular}

Figure 21: Optical prescription for the 3-element catadioptric design including the grating.
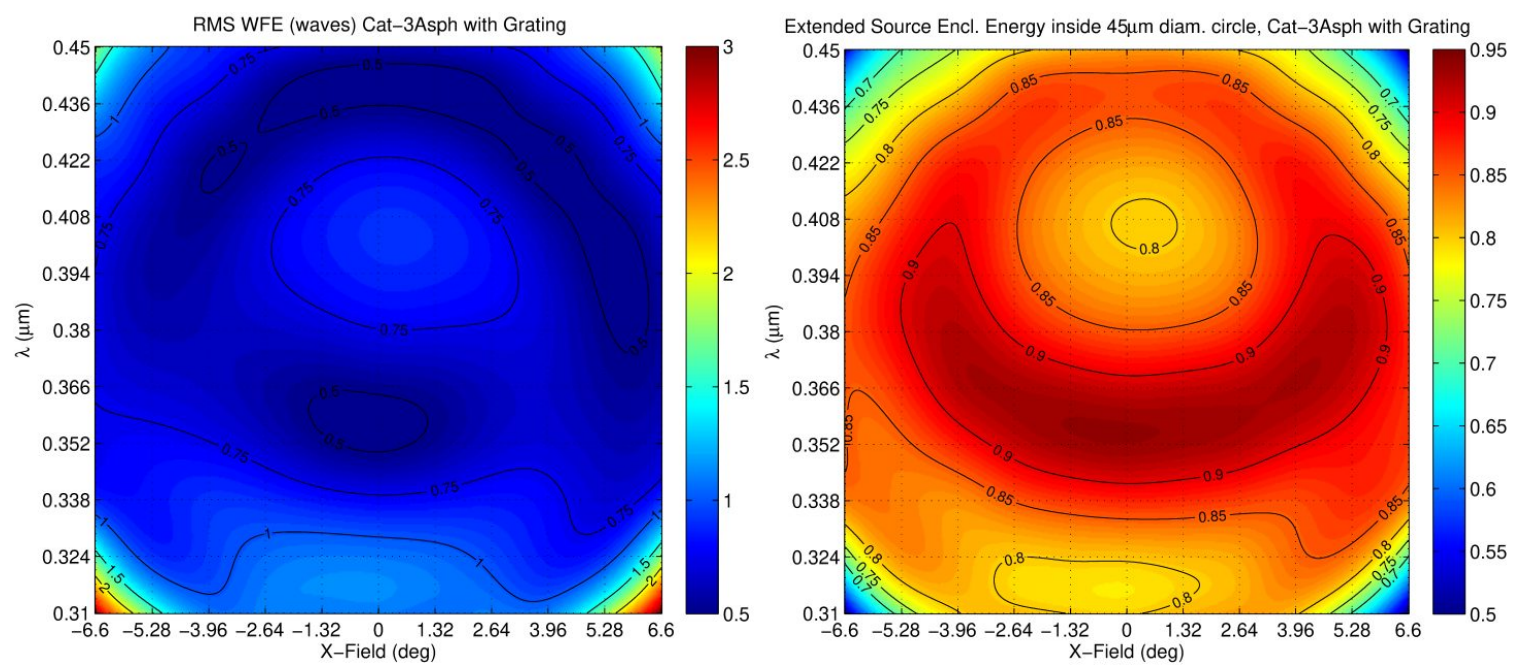

Figure 22: RMS WFE and extended source EE within $45 \mu \mathrm{m}$ diameter versus field and wavelength for the 3-element catadioptric design with grating.

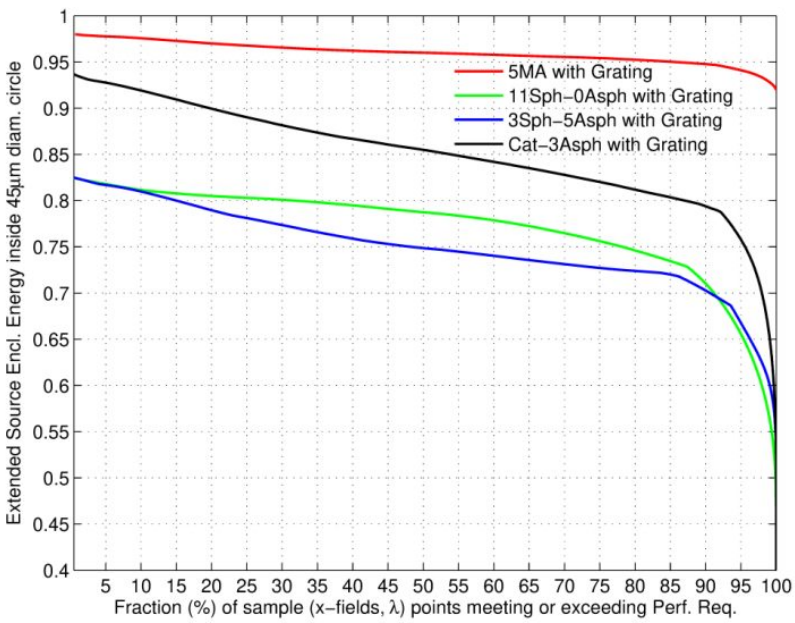

Figure 23: EE inside a 45micron diameter circle at the detector versus fraction of field and wavelength sample points meeting or exceeding a given performance level for the 11-element spherical lens with grating, the 8-element aspheric lens with grating, the 5MA with grating, and the 3 -element catadioptric design. 


\subsubsection{Tolerance analysis}

The tolerances listed in Table 1 were imposed on the catadioptric design. Four active compensation strategies were studied in 1,000 Monte Carlo simulation trials with uniform opto-mechanical error statistics:

- Active BFD adjustment,

- Active Pupil Relief adjustment,

- Active detector plane X-and Y-tilt adjustment.

Figure 24 shows Monte Carlo tolerance results, which indicate that the 3-element catadioptric design is robust against the opto-mechanical errors listed in Table 1. This design is therefore superior to the refractive and reflective designs investigated when performance is balanced against robustness to opto-mechanical errors.

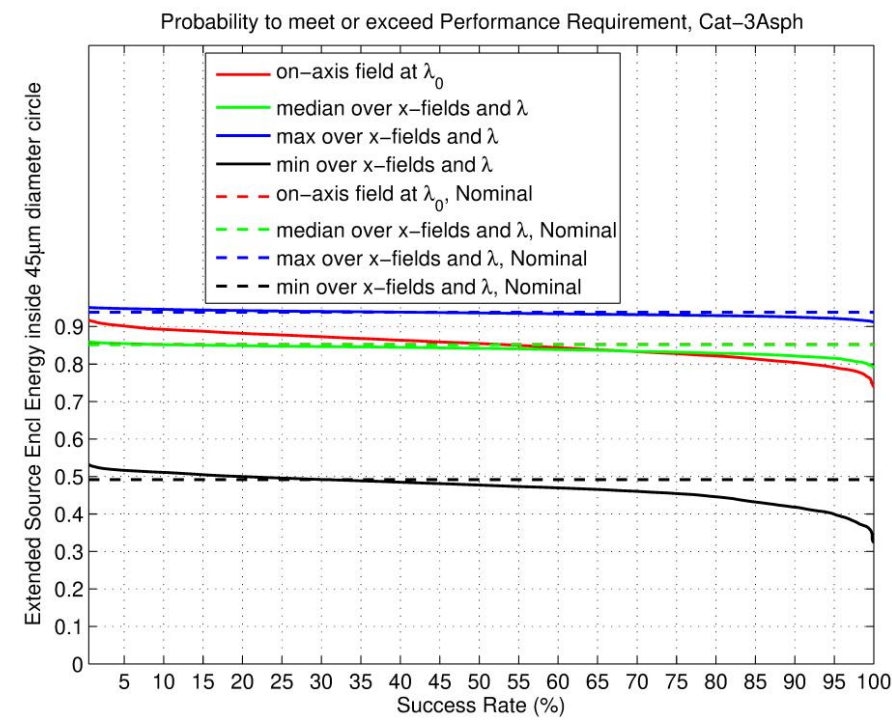

\begin{tabular}{|c|c|c|}
\hline \multicolumn{3}{|c|}{$\begin{array}{l}\text { Compensator statistics: } \\
\text { Thickness Surf } 8:\end{array}$} \\
\hline Nominal & : & 161.066979 \\
\hline Minimum & : & 155.858909 \\
\hline Maximum & : & 162.738036 \\
\hline Mean & : & 160.953741 \\
\hline Standard Deviation & : & 0.884169 \\
\hline \multicolumn{3}{|l|}{ Thickness Surf 14: } \\
\hline Nominal & : & -14.299155 \\
\hline Minimum & : & -14.611573 \\
\hline Maximum & : & -13.986230 \\
\hline Mean & : & -14.299148 \\
\hline Standard Deviation & : & 0.054736 \\
\hline \multicolumn{3}{|l|}{ Parameter 3 Surf 15: } \\
\hline Nominal & : & 0.000956 \\
\hline Minimum & : & -0.005262 \\
\hline Maximum & : & 0.006985 \\
\hline Mean & : & 0.000952 \\
\hline Standard Deviation & : & 0.001687 \\
\hline \multicolumn{3}{|c|}{ Parameter 4 Surf 15: } \\
\hline Nominal & : & 0.004018 \\
\hline Minimum & : & -0.014222 \\
\hline Maximum & : & 0.022384 \\
\hline Mean & : & 0.004022 \\
\hline Standard & : & 0.003594 \\
\hline
\end{tabular}

Figure 24: Monte Carlo tolerance results for the 3-element catadioptric camera with grating. 1,000 Monte Carlo trials were performed with uniform opto-mechanical error statistics. Success rate is defined as the probability over simulation trials to meet or exceed a given performance level. Right: compensator statistics for the 3-element catadioptric camera including the grating. The first group is pupil relief, the second is BFD and last two are image plane tilt about the $\mathrm{x}$ - and y-axis respectively.

\section{CONCLUSIONS}

In this paper, 2 refractive designs, 1 reflective freeform design, and 1 catadioptric design were investigated for the UV camera of the TMT fiber WFOS instrument concept based on first-order instrument parameters provided in August 2017. All 4 camera designs could easily be re-optimized for the latest fiber WFOS parameters if needed.

The camera full FoV along the spatial (X) direction is $13.2^{\circ}$ and $12.6^{\circ}$ along the dispersion (Y) direction, the EPD is $291 \mathrm{~mm}$ and the stop is external, the focal length is $392 \mathrm{~mm}$ (the paraxial focal ratio is $f / 1.35$ at the primary wavelength $\left.\lambda_{0}=0.38 \mu \mathrm{m}\right)$. The blue channel covers wavelengths ranging from $\lambda_{1}=0.31 \mu \mathrm{m}$ to $\lambda_{2}=0.45 \mu \mathrm{m}$. The spectrograph magnification is $m=-f_{\text {cam }} / f_{\text {col }}=-0.45$. Fibers are 100 microns in diameter, and the grating has a period equal to $v=1.475$ lines/micron, which leads to a $16.275 \mathrm{deg}$ grating tilt in Littrow configuration.

The top-level end-to-end performance requirement for the instrument is $90 \%$ extended source encircled energy (EE) inside a 45 micron diameter circle on the detector for a 100 micron diameter object (fiber core diameter), which for the chosen collimator focal length, corresponds to a fiber angular diameter of $23.65 \mathrm{arcsec}$. 
- Custom macros were written to compute in post-processing extended source enclosed energy and RMS WFE maps as a function of $\mathrm{x}$-fields (fibers in the pseudo-slit) and wavelengths. Data analysis and visualization was performed using Matlab.

- Nominal performance results are displayed in Figure 23. Both refractive designs provide $70 \%$ encircled energy inside a fiber over $90 \%$ of $x$-fields and wavelengths, whereas the 3 -element catadioptric camera is able to achieve $80 \%$ and the freeform 5MA 95\% encircled energy over the same percentage of $\mathrm{x}$-fields and wavelengths.

- Because of its speed, the camera is very sensitive to defocus: $\pm 20 \mu \mathrm{m}$ axial shift of the detector about its nominal position produces a $10 \%$ encircled energy loss. Similarly, \pm 0 .1deg detector tilt about its nominal position produces also a $10 \%$ encircled energy loss.

- A tolerance analysis was carried out for each design. Monte Carlo simulation results with high-precision optomechanical tolerances applied are displayed for the spherical lens in Figure 8, for the aspheric lens in Figure 13, for the 5MA in Figure 19, and for the 3-element catadioptric camera in Figure 24. For the tolerances analyzed (pending mechanical review), for a 95\% success rate (confidence level), the spherical lens exhibits a median extended source encircled energy over x-fields and wavelengths of only $30 \%$, the aspheric lens of only $20 \%$, the $5 \mathrm{MA}$ of only $40 \%$, and the 3-element catadioptric lens of $83 \%$. The 3-element catadioptric design, despite its $20-25 \%$ vignetting, is therefore superior to the refractive and reflective designs investigated when performance is balanced against robustness to opto-mechanical errors. Refinement of tolerances is needed after iteration with mechanical engineering and review of manufacturing capabilities. Although providing exquisite nominal performance, the 5MA design suffers from higher sensitivity to sag and tilt errors than the refractive and catadioptric designs. This can be understood in light of the fact that for a mirror, power is proportional to twice the sag (double pass effect), whereas for a lens the proportionality factor is the index minus one, which is about 4x smaller than for a mirror.

\section{ACKNOWLEDGEMENTS}

Luc Gilles is grateful to Jose Sasian for allowing him to work on this project as part of lens design coursework and independent study at the College of Optical Sciences at the University of Arizona, to Renate Kupke from the WFOS team for providing two previous refractive camera designs and for the opportunity to participate in the WFOS conceptual design, and to Andrew Rakich for discussions and pointing out the 3-element catadioptric solution.

\section{REFERENCES}

[1] https://www.ucolick.org/home/facilities/instruments/wfos.html

[2] "The Dark Energy Spectroscopic Instrument (DESI)", Proc. SPIE 9147, 91470S (2014), doi: 10.1117/12.2057105

[3] http://desi.lbl.gov/wp-content/uploads/2014/04/fdr-instrument-biblatex.pdf

[4] "MOONS: The multi-object optical and near-infrared spectrograph for the VLT", Proc. SPIE 9147, 91470N (2014), doi: $10.1117 / 12.2056012$

[5] http://titan.physx.u-szeged.hu/ fureszg/mtech/iii/JAS_grating_spectroscopy_theory_new.pdf

[6] C.Palmer "Diffraction grating handbook", $7^{\text {th }}$ edition, Newport Corp (2004)

[7] http://www.nikon.com/products/glass/lineup/materials/

[8] https://www.e2v.com/resources/account/download-datasheet/3828

[9] https://arxiv.org/ftp/arxiv/papers/1309/1309.6569.pdf

[10] N. Konidaris “MOBIE blue camera 140906d" (Oct 8, 2014) (available upon request).

[11] R.Bernstein and B.Bigelow "An optical design for a wide-field, optical spectrograph for TMT", Proc. SPIE 7014, doi: $10.1117 / 12.787297(2008)$.

[12] https:/www.optimaxsi.com/innovation/optical-manufacturing-tolerance-chart/

[13] M. Sanson "Understanding product cost versus performance through an in-depth system Monte Carlo analysis", Proc. SPIE 10377, 1037707 (2017), doi: 10.1117/12.2272494

[14] A.Rakich, "Four-mirror anastigmats, part 2: systems with useful first-order layouts and minimum complexity", Opt. Eng. 47, 033002 (2008). 
[15] J.Silny, E.D.Kim, L.G.Cook, E.M.Moskun and R.L.Patterson, "Optically fast, wide-field-of-view, five-mirror anastigmat imagers for remote sensing applications”, Proc. SPIE 8158 (2011), doi: 10.1117/12.893136

[16] https://www.slideshare.net/operacrazy/freeform-aspheric-talk

[17] https://www.lambdares.com/wp-content/uploads/support/oslo/oslo_releases/OSLOProgramReference.pdf

[18] T.Blalock, K.Medicus, J.DeGroote Nelson, "Fabrication of freeform optics", Proc. SPIE 9575 (2015), doi: $10.1117 / 12.2188523$

[19] S.DeFisher, G.Matthew and J.Ross, "New advancements in freeform optical metrology", Proc. SPIE 10179 (2017), doi: $10.1117 / 12.2269469$

[20] S.Mühlig, J.Siepmann, M.Lotz, A.Wiegmann, G.Blobel,S.Mika, A.Beutler and U.Nehse, "Current developments on optical asphere and freeform metrology", Proc. SPIE 9628 (2015), doi: 10.1117/12.2191247

[21] C.Pruss, G.B.Baer, J.Schindler, W.Osten, "Measuring aspheres quickly: tilted wave interferometry", Opt. Eng. 56(11) (2017), doi: 101117/1.OE.56.11.111713

[22] "Exporting Dutch mechatronics to China", Mikroniek 4 (2017).

[23]P.Mouroulis, "Optical design and engineering: lessons learned", Proc. SPIE 5865 (2005), doi: 10.1117/12.624548

[24]B.Delabre and E.Oliva, "A full silica F/0.95 compact, achromatic and athermalized camera compatible with $61 \mathrm{x}$ 61 mm detector", doi: 10.1117/12.2287567, IODC (2017).

[25] http://www.winlight-system.com/

[26] https://www.taylor-hobson.com/aboutus/overview/luphos 